\title{
Design framework of large-scale one-way electric vehicle sharing systems: A continuum approximation model
}

\author{
Xiaopeng $\mathrm{Li}^{\mathrm{a} *}$, Jiaqi Ma ${ }^{\mathrm{b}}$, Jianxun $\mathrm{Cui}^{\mathrm{c}}$, Amir Ghiasi ${ }^{\mathrm{a}}$ and Fang Zhou ${ }^{\mathrm{b}}$ \\ a. Department of Civil and Environmental Engineering, \\ University of South Florida, FL 33620 \\ b. Transportation Solutions and Technology Applications Division, \\ Leidos, Inc., VA 22101 \\ c. School of Transportation Science and Engineering, \\ Harbin Institute of Technology, Harbin, China 150001
}

\begin{abstract}
This paper proposes a Continuum Approximation (CA) model for design of a one-way Electrical Vehicle (EV) sharing system that serves a metropolitan area. This model determines the optimal EV sharing station locations and the corresponding EV fleet sizes to minimize the comprehensive system cost, including station construction investment, vehicle charging, transportation and vehicle balancing, under stochastic and dynamic trip demands. This is a very complex problem due to the NP-hard nature of location design, the large number of individual users, and the stochasticity and dynamics of generated trips. Further, the considerable charging time required by EVs distinguishes this problem from traditional car sharing problems where a vehicle is immediately available for pickup after being dropped at a station. We find that the CA approach can overcome these modeling challenges by decomposing the studied area into a number of small neighborhoods that each can be approximated by an Infinite Homogeneous Plane (IHP). We find that the system cost of an IHP is a unimodal function of the station service area size and can be efficiently solved in a sub-linear time by the bisection algorithm. Then integrating the solutions of all IHPs yields an approximate solution to the original heterogeneous area. With numerical experiments, we show that the CA solution is able to estimate the total system cost of the discrete counterpart solution very efficiently with good accuracy, even for large-scale heterogeneous problems. This implies that the proposed CA approach is capable of providing a near-optimum solution to the comprehensive design of a practical large-scale EV sharing system. With this model, we also conduct sensitivity analysis to reveal insights into how cost components and system design vary with the key parameter values. As far as the author's knowledge, this study is the first work that addresses design of an EV sharing system considering both longer-term location and fleet size planning and daily vehicle operations. The proposed CA model also extends the CA methodology literature from traditional location problems with stationary demand, single-facility based service to EV sharing problems considering dynamic demands, OD trips, and nonlinear vehicle charging times.
\end{abstract}

${ }^{*}$ Corresponding author. Tel: 813-974-0778, E-mail: xiaopengli@usf.edu. 


\section{Introduction}

The dominance of privately owned vehicles in North America, though providing incomparable mobility, flexibility and freedom to travel, actually imposes a huge challenge to sustainable transportation. The private auto mode constitutes over $83 \%$ of the total passenger trips in the US. Every year in the US, private vehicles are a major contributor to $17 \%$ household expenses on transportation, around $70 \%$ of the total petroleum consumption, and around $30 \%$ of green house gas emission (Bureau, 2014). In addition, personal vehicles, while being parked 23 hours a day (Litman, 2007), account for around 688,000 acres of land (Jakle and Sculle, 2004) and 25\% of urban surface occupancy $^{1}$, which further exacerbates the already congested urban traffic (Axhausen et al., 1994). While public transit is able to overcome these defects, this mode is not utilized very well in many low-density areas in the US due to its disadvantages in mobility and accessibility (Sinha, 2003).

Car sharing is an alternative to private car ownership such that a group of people collectively own or use a number of spatially distributed vehicles (Cooper et al., 2000). Car sharing has been recognized as the missing link to sustainable transportation that integrates flexibility, mobility and accessibility from private vehicles and economy and sustainability from public transits (Britton, 2000). Over the past decade in North America, the number of shared vehicles have increased from under 700 to over 15,000, and the people who use this service have grown from 16,000 to over a million (Shaheen and Cohen, 2013). Successful businesses across the world include Zipcar (http://www.zipcar.com/) and JustShareIt (http://www.justshareit.com/) in the US, Autolib (https://www.autolib.eu/en/) in France, City Car Club (http://www.citycarclub.co.uk/) in the UK, Greenwheels (https://www.greenwheels.com) in the Netherlands, Stadtmobil (http:// www.stadtmobil.de) in Gemany, and Zoom (http://www.zoomcar.com/) in India. As this trend continues and car sharing evolves from a niche service to a major transportation mode with high spatial accessibility, car sharing holds promise for a future sustainable transportation system with high vehicle utilization rates, minimum land occupancy, significant cost savings, and substantial environmental and social benefits (Millard-Ball, 2005; Nobis, 2006).

The environmental and social benefits of car sharing can be further enhanced by using electric vehicles (EVs) (Green, 2009). EVs reduce our dependence on depletable fossil fuels, produce zero tailpipe emissions and thus bring minimum pollution to urban areas. Even considering the life-cycle emissions generated from the corresponding electricity plants, the overall impact to the air quality is much less than that from internal combustion engine (ICE) vehicles (Wang et al., 2005). The social and environmental benefits will be further magnified if renewable energy sources are used to power EVs. In addition, on the end user side, unit-distance cost of an EV is significantly lower than that of an ICE vehicle (Valdes-Dapena, 2008), and the fixed vehicle cost is steadily decreasing as the battery technologies evolve and massive production brings economies of scale (Vlasic, 2013). The adoption of EVs, though slow for private vehicles (e.g., due to range anxiety), is expected to become prevalent if EVs and car sharing can be well integrated (King et al., 2013).

Despite the high expectations of the role of EV sharing in future transportation, there is not yet a comprehensive design framework on how to economically deploy an EV sharing system that is able to provide reliable service to stochastic trip demands in an urban area. This paper proposes a continuum approximation (CA) model for determining the optimal configuration of such an EV sharing system, including the location of car sharing stations and the corresponding vehicle inventories at these stations under stochastic demand and EV charging constraints. This optimal

\footnotetext{
${ }^{1}$ http://oldurbanist.blogspot.com/2011/12/we-are-25-looking-at-street-area.html.
} 
configuration shall minimize the total system life-time cost while assuring the service reliability to stochastic one-way trip demands. We analyze the structure of the elemental cost function in the CA model and find an elegant unimodal property that assures the superior computational performance. This enables us to solve its optimal value with a very fast sub-linear time bisection search algorithm. Numerical experiments show that this model can solve a large-scale problem instance to a near-optimum solution very efficiently. We apply this model to different city areas and draw interesting managerial insights into the optimal system design and the total system cost. Note that this paper studies car sharing without car-pooling (also known as ride sharing) and thus each vehicle only serves no more than one trip at a time.

\section{Literature Review}

The proposed research is built upon the existing studies on classic facility location problems, EV infrastructure planning, and car sharing system design. Daskin (1995) and Drezner (2002) provided comprehensive reviews of fundamental facility location problems (e.g., covering, median, center, fixed-charge location problems). In these fundamental models, each customer is basically assigned to one facility with an operational cost increasing with the distance between them, and the design goal is to determine the best locations to build facilities to balance the trade-off between facility investment and operation costs. These fundamental models have been extended to joint design with other system elements, e.g., commodity inventory at each facility (Shen et al., 2003; Chen et al., 2011), which is particularly relevant to this study where the vehicle storage needs to be jointly determined with car sharing station location. A typical way to approach location problems is to discretize the space and solve the location design with integer programming (IP) techniques. However, location problems are in general NP-hard, and the IP approach may suffer from excessive computational burdens. To overcome this challenge, Daganzo and Newell (1986) developed a continuum approximation (CA) model that decomposes the complex location problem into a set of fairly simple homogeneous problems that each approximates a neighborhood of the original problem. CA can efficiently solve NP-hard network problems such as facility location (Ouyang 2006; Cui et al. 2010; Li and Ouyang 2010; Carlsson et al. 2013), vehicle routing (Langevin 1996; Ouyang et al. 2014) and hub-and-spoke networks (Campbell, 1993a; Carlsson and Jia, 2013). It has been applied to air networks (Hall, 1989), one-to-many distribution (Campbell, 1993b), demand uncertainty (Daganzo and Erera, 1999), supply disruptions (Li and Ouyang, 2010), integrated inventory-location (Tsao et al., 2012), and facility competition (Wang and Ouyang, 2013). Ouyang et al. (2015) recently innovated the CA concept to devise a compact continuous model that efficiently solves the complex user-equilibrium traffic assignment problem under certain settings. Although CA essentially falls in general heuristic algorithms, it is rooted in analytical modeling and therefore is able to yield elegant insights into the problem structure. Further, analysis shows that CA can yield a location design very close to the true optimum (Daganzo, 2005).

A handful of recent studies investigated location of refueling stations for alternative fuel vehicles (AFV). Refueling location research stems from flow covering problem that aims to maximally intercept traffic flows with minimum stations (Berman et al., 1992; Hodgson, 1990). These studies were later extended to refueling location problems that consider limited vehicle travel range (Kuby and Lim, 2005; Kuby et al., 2009; Wang and Lin, 2009), limited fueling capacity (Upchurch et al., 2009), deviation paths (Berman et al., 1995; Huang et al., 2015), integration with vehicle scheduling (Nourbakhsh and Ouyang, 2010), and different battery capacities (Nie and Ghamami, 2013). As an 
alternative to time-consuming charging, battery exchange is a fast mode of EV fueling, and battery exchange station location problems were investigated by Pan et al. (2010) and Mak et al. (2013). Other studies further expanded the scope of the problem to include power grid configurations and seek optimal design of integrated vehicle-to-grid (V2G) systems (Pan et al., 2010; He et al., 2013, 2014). In addition to mathematical programming, data mining (e.g., hierarchical clustering by Ip et al. (2010)) and simulation-based optimization (e.g., agent based simulation in Sweda and Klabjan (2011)) were applied to solve similar EV infrastructure planning problems.

Although the practice of car sharing can be dated to 1940s in Europe and to 1980s in North American (Shaheen and Cohen, 2013), quantitative research on design of a car sharing system is relatively scarce. Early car sharing systems only served a limited number of members and were regarded as niche markets (Millard-Ball, 2005). Shaheen et al. (1998) qualitatively argued that a dense network and variety of vehicles contribute to the success of a car sharing business. Based on the existing fleet assignment models (e.g., Du and Hall (1997)), several modeling approaches were adopted to analyze operations of a car sharing system. Simulation models (Barth and Todd, 1999; Uesugi et al., 2007; Ciari et al., 2008) were built to analyze the sensitivity of the system cost and the service quality to system parameters such as fleet size and vehicle relocation. A queuing model was proposed by George and Xia (2011) to determine the optimal fleet size of shared vehicles. Mathematical programming models were developed to determine optimal vehicle relocation, considering stationary demand (Chauvet et al., 1997), dynamic demand (Fan et al., 2008; Kek et al., 2009), EV charging delay Bruglieri et al. (2014) and free-floating sharing (Pal and Zhang, 2016). Only a couple of recent studies made a step further and addressed the location of car sharing stations using discrete integer programming models (Correia and Antunes, 2012; Kumar and Bierlaire, 2012). Most of the existing studies on car-sharing only focus on two-way car sharing problems that require a user to return a vehicle at the same place where she picks up this vehicle and thus have a relatively simple problem structure. One-way car sharing systems, where a customer can drop a vehicle at any car sharing facility, have much more complicated operations and are not as well investigated.

As electric cars become increasingly popular in car sharing service due to their environmental and economic advantages (Green, 2009), there is an imperative need for a comprehensive design framework for an EV car sharing system. This proposed study aims to bridge this gap between the growth of the EV car sharing industry and the lack of system design methodologies. This study makes a number of contributions to the existing literature. First, we propose a car sharing system design framework that considers both planning of station locations and fleet sizes and operations of EV dispatches and rebalancing under uncertain dynamic demand. Second, the vehicle operation component incorporates a realistic nonlinear charging process of EVs at the charging stations (collocated with car sharing facilities), which allows us to investigate the impact of charging capability of EVs to the performance of the EV car sharing system. Third, this design framework is modeled with a CA approach that has favorable computational tractability due to the unimodality of its elemental cost function while preserving reasonable approximation accuracy. Finally, we construct real-world case studies with realistic traffic data of various scales to test the model and reveal managerial insights. 


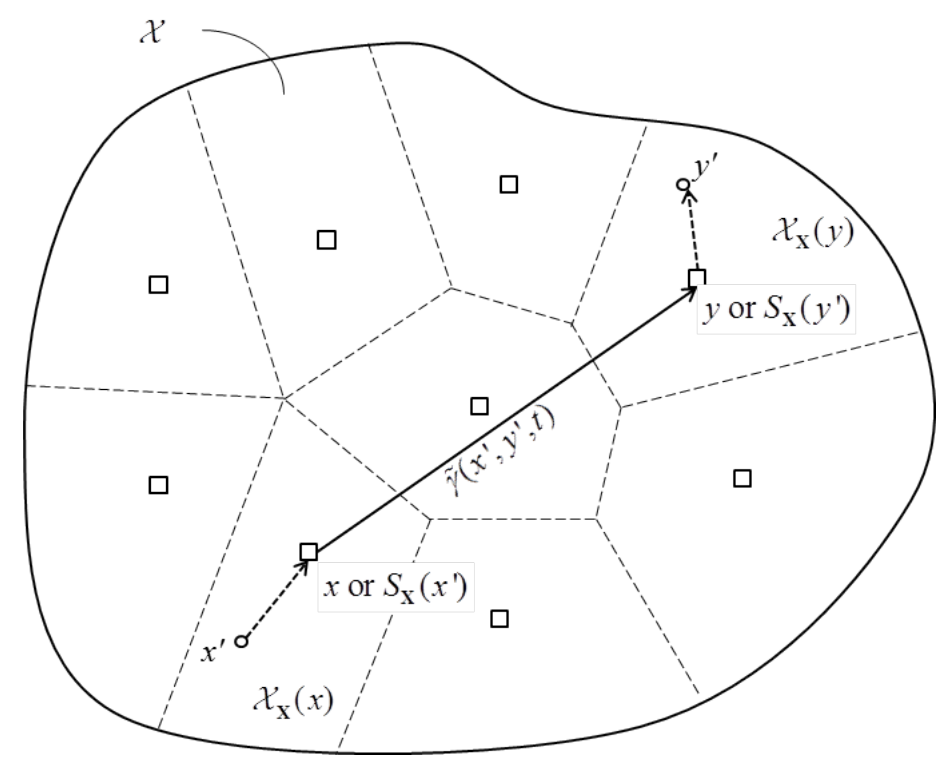

Figure 1: Illustration of the continuous space.

\section{General Problem Formulation}

For the convenience of the readers, some key symbols are listed in the Appendix. We consider a two-dimensional space $\mathcal{X} \subseteq \mathbb{R}^{2}$ where we plan to build a number of $N$ EV sharing facilities at locations $\mathbf{x}:=\left\{x_{1}, x_{2}, \ldots, x_{N}\right\} \subseteq \mathcal{X}$. We assume that these locations shall be relatively dense so that every traveler can relatively easily access the service. We assume that building a facility at location $x \in \mathcal{X}$ takes a certain construction cost, e.g., due to the construction of the parking space and charging stations. For the convenience of the calculation, we let $f(x)$ denote construction cost at location $x$ (prorated to a unit operational cycle). The the total construction cost of this system is simply the summation of the construction costs across all built facilities, i.e.,

$$
\bar{C}_{f}:=\sum_{x \in \mathbf{x}} f(x)
$$

Once all facilities are built, travelers will share EVs at these facilities to complete their trips. As illustrated in Figure 1, space $\mathcal{X}$ is essentially divided into $|\mathbf{x}|$ partitions by the Voronoi Tessellation (Okabe et al., 2009) with seeds $\mathbf{x}$. We denote the set of locations in the partition with seed $x$ by $\mathcal{X}_{\mathbf{x}}(x)$, i.e.,

$$
\mathcal{X}_{\mathbf{x}}(x):=\left\{x^{\prime} \mid\left\|x^{\prime}-x\right\| \leq\left\|x^{\prime}-y\right\|, \forall y \neq x \in \mathbf{x}\right\}, \forall x \in \mathbf{x},
$$

and we denote the seed for any location $x^{\prime} \in \mathcal{X}$ by $S_{\mathbf{x}}\left(x^{\prime}\right)$, i.e.,

$$
S_{\mathbf{x}}\left(x^{\prime}\right):=\arg \min _{x \in \mathbf{x}}\left\|x^{\prime}-x\right\|, \forall x^{\prime} \in \mathcal{X} .
$$

Note that every traveler originated in $\mathcal{X}_{\mathbf{x}}(x)$ always goes to facility $x$ to pick up a car since $x$ is the closest facility to her. We consider an operation paradigm that repeats with an operational cycle of length $\bar{t}$ (e.g., a day), and therefore we only describe operations during one cycle period $[0, \bar{t}$. We assume that the unit-area trip rate from any $x^{\prime} \in \mathcal{X}_{\mathbf{x}}(x)$ to any $y^{\prime} \in \mathcal{X}_{\mathbf{x}}(y), \forall x, y \in \mathbf{x}$ (from a 
unit area in the neighborhood of $x^{\prime}$ to a unit area in the neighborhood in $y^{\prime}$ ) that departs from $x$ at time $t$ is a random variable $\gamma\left(x^{\prime}, y^{\prime}, t\right)$ following a probability distribution with a mean of $\bar{\gamma}\left(x^{\prime}, y^{\prime}, t\right)$ and a standard deviation of $\hat{\gamma}\left(x^{\prime}, y^{\prime}, t\right)$. We assume that the $\gamma\left(x^{\prime}, y^{\prime}, t\right)$ values are independent both temporally and spatially, and $\gamma\left(x^{\prime}, y^{\prime}, t\right)=0$ if $\left\|x^{\prime}-y^{\prime}\right\|<\underline{d}$ where $\underline{d}$ is the minimum distance for a trip to use a car instead of a direct walking. The distance between two points in the same partition is usually less than $\underline{d}$. Further, we assume that every traveler has a fixed EV pickup time regardless of facility location design $\mathbf{x}$ (which may affect partition sizes). This assumption is reasonable for our problem if any of the following conditions holds: (i) a traveler intends to book a vehicle at a fixed time to balance the earliness from the origin and the lateness at the destination regardless of partition sizes, (ii) every partition is relative small and the walking time is negligible, (iii) $\gamma\left(x^{\prime}, y^{\prime}, t\right)$ does not vary too much with time and thus the $\gamma\left(x^{\prime}, y^{\prime}, t\right)$ and $\gamma\left(x^{\prime}, y^{\prime}, t+\delta t\right)$ are almost identical for a small walking time deviation $\delta t$ due to partition size changes. With this assumption, trip rate $\gamma\left(x^{\prime}, y^{\prime}, t\right)$ is not affected by the location design of $\mathbf{x}$ and thus can be regarded as an external input.

To implement a trip from origin $x^{\prime}$ to destination $y^{\prime}$, a traveler will walk to EV sharing facility $S_{\mathbf{x}}\left(x^{\prime}\right)$ and pick up a fully-charged EV there. Then she will drive to facility $S_{\mathbf{x}}\left(y^{\prime}\right)$, drop off the car there and walk to $y^{\prime}$. We assume that the walking distance between any two points $x^{\prime}$ and $x$ in $\mathcal{X}$ is estimated as the product of the Euclidean distance between these two location $\left\|x^{\prime}-x\right\|$ and an inflation factor $\eta>1$ that captures the additional distance due to turns and curves on the travel path (Love and Morris, 1979; Qureshi et al., 2002). Let $w$ denote the unit-distance walking cost, then the expected total walking cost can be formulated as,

$$
\begin{aligned}
\bar{C}_{w}: & =w \eta \mathbf{E} \int_{t \in[0, \bar{t}], x, y \in \mathcal{X}} \gamma(x, y, t)\left(\left\|x-S_{\mathbf{x}}(x)\right\|+\left\|y-S_{\mathbf{x}}(y)\right\|\right) \\
& =w \eta \int_{t \in[0, \bar{t}], x, y \in \mathcal{X}} \bar{\gamma}\left(\left\|x-S_{\mathbf{x}}(x)\right\|+\left\|y-S_{\mathbf{x}}(y)\right\|\right)
\end{aligned}
$$

To reliably serve the stochastic travel demands, each facility $x$ holds a number of $I(x)$ EVs at the beginning time 0. Obviously, the higher the value of $I(x)$, the less likely the corresponding partition $\mathcal{X}_{\mathbf{x}}(x)$ runs out of EVs, and the higher the service reliability is. In order to quantitatively determine the $I(x)$ value in partition $\mathcal{X}_{\mathbf{x}}(x)$ to achieve certain service reliability, we need to investigate $\mathrm{EV}$ departure and arrival processes in each partition. We denote the cumulative departure count of EVs at facility $x \in \mathrm{x}$ at up to time $t$ by $\lambda(x, t)$, which can be formulated as the cumulative outbound trip demand in partition $\mathcal{X}_{\mathbf{x}}(x)$ by time $t$,

$$
\lambda(x, t):=\int_{t^{\prime}=0}^{t} \int_{x^{\prime} \in \mathcal{X}_{\mathbf{x}}(x), y^{\prime} \in \mathcal{X}} \gamma\left(x^{\prime}, y^{\prime}, t^{\prime}\right) d y^{\prime} d x^{\prime} d t^{\prime}, \forall x \in \mathbf{x}, t \in[0, \bar{t}] .
$$

Since $\gamma\left(x^{\prime}, y^{\prime}, t^{\prime}\right)$ values are non-negative independent Poisson random variables across time and space, $\lambda(x, t)$ at certain location $x$ and time $t$ shall also follow the Poisson distribution with a relatively high mean value. Therefore, with the central limit theory and the properties of the Poisson distributions (see https://en.wikipedia.org/wiki/Poisson_distribution, which states that the Poisson distribution with a mean greater than 10 can be reasonably well approximated by the normal distribution), $\lambda(x, t)$ can be well approximated with a random variable following the normal distribution with mean $\bar{\lambda}(x, t)$ and standard deviation $\hat{\lambda}(x, t)$, where

$$
\bar{\lambda}(x, t):=\int_{t^{\prime}=0}^{t} \int_{x^{\prime} \in \mathcal{X}_{\mathbf{x}}(x), y^{\prime} \in \mathcal{X}} \bar{\gamma}\left(x^{\prime}, y^{\prime}, t^{\prime}\right) d y^{\prime} d x^{\prime} d t^{\prime}, \forall x \in \mathbf{x}, t \in[0, \bar{t}],
$$


and

$$
\hat{\lambda}(x, t):=\left(\int_{t^{\prime}=0}^{t} \int_{x^{\prime} \in \mathcal{X}_{\mathbf{x}}(x), y^{\prime} \in \mathcal{X}} \hat{\gamma}\left(x^{\prime}, y^{\prime}, t^{\prime}\right)^{2} d y^{\prime} d x^{\prime} d t^{\prime}\right)^{0.5}, \forall x \in \mathbf{x}, t \in[0, \bar{t}] .
$$

Then we will formulate the cumulative arrival count of EVs at facility $x$. An EV depending on its battery status may be able to travel a distance range varying between 0 (when the battery is fully discharged) and $\bar{s}$ (when it is fully charged). We call this distance range the state of charge (SOC) of this EV. Let $H(s)$, which can be an arbitrary increasing function of $s$, denote the time to charge an EV at $\mathrm{SOC} \bar{s}-s$ to full SOC $\bar{s}$. A realistic function $H(s)$ is likely nonlinear, and it often takes a longer time to charge a unit SOC when the current SOC is already high (Chen, 2007). For simplicity of operations, we assume that a car sharing facility has sufficient charging capacity such that an inbound EV is connected to a charger as soon as it is dropped off, and an outbound EV is available for pickup only when it is fully charged (or its SOC equals $\bar{s})^{2}$. Since the EV sharing system only needs to satisfy the customer demand most of the time but not all the time (see the service reliability in Equations (7) and (8)), each facility actually only needs a finite charing capacity. We assume that an EV always travels at a constant speed $v$. Then an EV departing from facility $y$ at time $t$ won't be available at $x$ until time $t+\frac{\eta}{v}\|x-y\|+H(\eta\|x-y\|)$, where $\frac{\eta}{v}\|x-y\|$ is the EV travel time from $y$ to $x$ and $H(\eta\|x-y\|)$ is the time to charge this EV from its residual SOC $\bar{s}-\eta\|x-y\|$ upon its arrival at $x$ to the full level $\bar{s}$. Therefore, a combined trip from $y^{\prime} \in \mathcal{X}_{\mathbf{x}}(y)$ to $x^{\prime} \in \mathcal{X}_{\mathbf{x}}(x)$ needs to take off from $y$ before time $t^{\prime}-\frac{\eta}{v}\|x-y\|-H(\eta\|x-y\|)$ in order to have this EV available at facility $x$ by time $t^{\prime}$. Then the cumulative count of the available EVs among all arrival EVs (or "cumulative arrival count" for short) at facility $x$ by time $t$ can be formulated as follows.

$$
\begin{aligned}
\mu(x, t): & \left.=\sum_{y \neq x \in \mathbf{x}} \int_{t^{\prime}=0}^{t} \int_{x^{\prime} \in \mathcal{X}_{\mathbf{x}}(x)} \int_{y^{\prime} \in \mathcal{X}_{\mathbf{x}}(y)} \gamma\left(y^{\prime}, x^{\prime}, t^{\prime}-\frac{\eta}{v}\|x-y\|-H(\eta\|x-y\|)\right)\right) d y^{\prime} d x^{\prime} d t^{\prime} . \\
& =\int_{t^{\prime}=0}^{t} \int_{x^{\prime} \in \mathcal{X}_{\mathbf{x}}(x)} \int_{y^{\prime} \in \mathcal{X} \backslash \mathcal{X}_{\mathbf{x}}(x)} \gamma\left(y^{\prime}, x^{\prime}, t^{\prime}-\frac{\eta}{v}\left\|x-S_{\mathbf{x}}\left(y^{\prime}\right)\right\|-H\left(\eta\left\|x-S_{\mathbf{x}}\left(y^{\prime}\right)\right\|\right)\right) d y^{\prime} d x^{\prime} d t^{\prime}
\end{aligned}
$$

Again, $\mu(x, t)$ at specific location $x$ and time $t$ follows the Poisson distribution and most of the time it has a relatively large mean, and thus it approximately follows $\mathcal{N}(\bar{\mu}(x, t), \hat{\mu}(x, t))$, where

$$
\bar{\mu}(x, t):=\int_{t^{\prime}=0}^{t} \int_{x^{\prime} \in \mathcal{X}_{\mathbf{x}}(x)} \int_{y^{\prime} \in \mathcal{X}} \bar{\gamma}\left(y^{\prime}, x^{\prime}, t^{\prime}-\frac{\eta}{v}\left\|x-S_{\mathbf{x}}\left(y^{\prime}\right)\right\|-H\left(\eta\left\|x-S_{\mathbf{x}}\left(y^{\prime}\right)\right\|\right)\right) d y^{\prime} d x^{\prime} d t^{\prime},
$$

and

$$
\hat{\mu}(x, t):=\left(\int_{t^{\prime}=0}^{t} \int_{x^{\prime} \in \mathcal{X}_{\mathbf{x}}(x)} \int_{y^{\prime} \in \mathcal{X}} \hat{\gamma}\left(y^{\prime}, x^{\prime}, t^{\prime}-\frac{\eta}{v}\left\|x-S_{\mathbf{x}}\left(y^{\prime}\right)\right\|-H\left(\eta\left\|x-S_{\mathbf{x}}\left(y^{\prime}\right)\right\|\right)\right)^{2} d y^{\prime} d x^{\prime} d t^{\prime}\right)^{0.5} .
$$

In the above equations, it appears that $\mu(x, t)$ depends on locations of facilities other than $x$, which may result in a very hard hub-and-spoke type of complexity in this location design problem.

\footnotetext{
${ }^{2}$ Such operation also reduces the charging depth and consequentially prolongs the battery life (http://batteryuniversity.com/learn/article/how_to_prolong_lithium_based_batteries).
} 
Fortunately, we find out that $\|x-y\|$ can be approximated with $\left\|x^{\prime}-y^{\prime}\right\|$ without much affecting $\mu(x, t)$ values (and thus the dependence of $\mu(x, t)$ on other facilities can be decoupled), due to the following reasons. First, $\left\|x^{\prime}-y^{\prime}\right\|$ shall be not too much different from $\|x-y\|$ if $x$ and $y$ are relatively distant compared to the radii of $\mathcal{X}_{\mathbf{x}}(y)$ and $\mathcal{X}_{\mathbf{x}}(x)$, which is the case for most OD pairs if locations $\mathbf{x}$ is relatively dense. More importantly, if both $\mathcal{X}_{\mathbf{x}}(y)$ and $\mathcal{X}_{\mathbf{x}}(x)$ are close to a regular shape (e.g., a circle or a regular hexagon), $\|x-y\|$ is almost identical to the average travel distance from a point in $\mathcal{X}_{\mathbf{x}}(y)$ to one in $\mathcal{X}_{\mathbf{x}}(x)$, and thus replacing $\|x-y\|$ with $\left\|x^{\prime}-y^{\prime}\right\|$ in equation (3) little affects the value of $\mu(x, t)$. Therefore, $\bar{\mu}(x, t)$ and $\hat{\mu}(x, t)$ can be reformulated as

$$
\bar{\mu}(x, t)=\int_{t^{\prime}=0}^{t} \int_{x^{\prime} \in \mathcal{X}_{\mathbf{x}}(x)} \int_{y^{\prime} \in \mathcal{X}} \bar{\gamma}\left(y^{\prime}, x^{\prime}, t^{\prime}-\frac{\eta}{v}\left\|x^{\prime}-y^{\prime}\right\|-H\left(\eta\left\|x^{\prime}-y^{\prime}\right\|\right)\right) d y^{\prime} d x^{\prime} d t^{\prime},
$$

and

$$
\hat{\mu}(x, t):=\left(\int_{t^{\prime}=0}^{t} \int_{x^{\prime} \in \mathcal{X}_{\mathbf{x}}(x)} \int_{y^{\prime} \in \mathcal{X}} \hat{\gamma}\left(y^{\prime}, x^{\prime}, t^{\prime}-\frac{\eta}{v}\left\|x^{\prime}-y^{\prime}\right\|-H\left(\eta\left\|x^{\prime}-y^{\prime}\right\|\right)\right)^{2} d y^{\prime} d x^{\prime} d t^{\prime}\right)^{0.5} .
$$

With the definitions of $\lambda(x, t)$ and $\mu(x, t)$, the net cumulative departure count can be obtained as

$$
\theta(x, t):=\lambda(x, t)-\mu(x, t), \forall x \in \mathbf{x}, t \in[0, \bar{t}] .
$$

The value of $\theta(x, t)$, if positive, indicates the number of EVs needed from the initial fleet of size $I(x)$ to satisfy all departure trips by time $t$ at facility $x$ without incurring any delay. Since $\lambda(x, t)$ and $\mu(x, t)$ approximately follow the normal distribution, $\theta(x, t)$ at specific location $x$ and time $t$ shall be also well approximated with a normal random variable with an expectation of $\bar{\theta}(x, t):=$ $\bar{\lambda}(x, t)-\bar{\mu}(x, t)$ and a standard deviation of $\hat{\theta}(x, t):=\left(\hat{\lambda}(x, t)^{2}+\hat{\mu}(x, t)^{2}\right)^{0.5}$. Note that standard deviation $\hat{\theta}(x, t)$ increases with time $t$.

Theoretically, $I(x)$ has to be infinity if we absolutely require all departure trips at facility $x$ to be immediately satisfied in any scenario. A common practice is that $I(x)$ is instead set to a reasonable finite value to assure that most departure demands are served without delay. Based on the distribution of $\theta(x, t)$, we can determine $I(x)$ with the following two service reliability criteria.

Fixed-Time Availability (FTA): For a facility $x$, select a fixed time point $\hat{t}$ as a reference point (e.g., a morning peak hour point, which has a relatively high probability of EV outage). This reliability requirement imposes that the probability that EVs are available at facility $x$ at time $\hat{t}$ is no less than a high percentage $\alpha$ (e.g., 95\%), i.e., $\operatorname{Pr}[\theta(x, \hat{t}) \leq I(x)] \geq \alpha$. Since $\theta(x, \hat{t}) \sim \mathcal{N}\left(\bar{\theta}(x, \hat{t}), \hat{\theta}(x, \hat{t})^{2}\right)$, the minimum $I(x)$ to assure this service reliability is

$$
I^{F}(x):=\left[\bar{\theta}(x, \hat{t})+\Phi^{-1}(\alpha) \hat{\theta}(x, \hat{t})\right]^{+}
$$

where $\Phi(\cdot)$ is the cumulative distribution function (CDF) of a standard normal distribution, $\Phi^{-1}(\cdot)$ is its inverse, and operator $[\cdot]^{+}=\max \{0, \cdot\}$ that assures the initial inventory to be nonnegative. 
Peak-Time Availability (PTA): This reliability criterion is similar to FTA except that the time point of the highest car outage probability is examined. Note that this peak time may depend on location design $\mathbf{x}$. The minimum $I(x)$ to assure this reliability measure is

$$
I^{P}(x):=\max _{t \in[0, \bar{t}]} \bar{\theta}(x, t)+\Phi^{-1}(\alpha) \hat{\theta}(x, t)
$$

Note that $I^{P}(x)$ is always non-negative since $\bar{\theta}(x, 0)+\Phi^{-1}(\alpha) \hat{\theta}(x, 0)=0$, and thus the nonnegativity operator is not needed. Once the value of $I(x)$ is determined, we can formulate the cost needed to hold these EVs. We assume that each station $x$ is responsible for the holding costs of all $I(x)$ EVs in its initial fleet. Let $h(x)$ denote the cost needed (e.g., for purchase and maintenance) to hold an EV at a location $x \in \mathcal{X}$ over an operational cycle. Then the total EV holding cost will be

$$
\bar{C}_{h}:=\sum_{x \in \mathbf{x}} h(x) I(x)
$$

At time $\bar{t}$, which is the end of this period and the start of the next operation period, the number of EVs at facility $x$ is different from the initial inventory $I(x)$ by $\theta(x, \bar{t})^{3}$. If $\theta(x, \bar{t})$ is positive, we need to bring EVs from nearby facilities to rebalance the car inventory at facility $x$ back to its initial level $I(x)$. The expected inventory that needs to be rebalanced at facility $x$ can be calculated by

$$
I_{\bar{t}}(x):=\int_{0}^{\infty} z d \Phi\left(\frac{z-\bar{\theta}(x, \bar{t})}{\hat{\theta}(x, \bar{t})}\right) .
$$

The exact rebalancing problem itself could be rather complex and it could involve complex routing if vehicles are transported in bundles by trucks (similar to rebalancing in bike sharing, e.g., Chemla and Calvo (2013), Regue and Recker (2014) and Pal and Zhang (2016),). Our problem instead considers that each EV is rebalanced individually (Smith et al., June, 2013). For simplicity, we assume that the cost of moving an EV is dominated by a fixed handling cost $b$ regardless of the moving distance, which is reasonable since the distances between neighboring stations are relatively short given that the EV sharing facilities are relatively dense. Then the expected rebalancing cost in a operational cycle is

$$
\bar{C}_{b}:=\sum_{x \in \mathbf{x}} b I_{\bar{t}}(x)
$$

After the rebalancing is done, every facility resets its car inventory back to the initial level. This way, the following cycle repeats statistically identical operations (i.e., all parameters and variables shall follow the same distributions as those in the previous cycle). This confirms that it is sufficient to just examine operations in one cycle $[0, \bar{t}]$.

Since $\|y-x\|$ is nearly identical to the average driving distance between two points in $\mathcal{X}_{\mathbf{x}}(y)$ and $\mathcal{X}_{\mathbf{x}}(x)$, the total driving distance would be approximately

$$
\bar{C}_{d}:=\int_{0}^{\bar{t}} \int_{x^{\prime}, y^{\prime} \in \mathcal{X}} \gamma\left(x^{\prime}, y^{\prime}, t\right) d x^{\prime} d y^{\prime} d t
$$

\footnotetext{
${ }^{3}$ We assume that $\mu(x, t)$ is low around time $\bar{t}$, and thus $\theta(x, \bar{t})$ is a good approximation of the difference between the actual EV count at facility $x$ at time $\bar{t}$ and the initial inventory $I(x)$.
} 
The system design problem is to determine facility locations $\mathbf{x}$ (including the number of facilities $|\mathbf{x}|$ and their locations), and the corresponding EV inventory levels $\mathbf{I}:=\left\{I\left(x_{1}\right), I\left(x_{2}\right), \cdots, I\left(x_{N}\right)\right\}$ to minimize the expected total system cost. Note that driving cost $\bar{C}_{d}(12)$ is determined by the external travel demands $\left\{\gamma\left(x^{\prime}, y^{\prime}, t\right)\right\}$ and independent of system $\mathbf{x}$ and $\mathbf{I}$. Thus we will not consider $\bar{C}_{d}$ in system design. Then the system design problem can be formulated as

$$
\min _{\mathbf{x}, \mathbf{I}} \bar{C}:=\bar{C}_{f}+\bar{C}_{w}+\bar{C}_{h}+\bar{C}_{b}
$$

For a realistic urban area with a large number of trips, this problem is still a very complex optimization problem that is hard to be solved to the exact optimum. This study only focuses on settings where every parameter varies relatively slowly across space $\mathcal{X}$. With this slowly-varying property, we can adapt an efficient continuum approximation (CA) approach to solve this problem to an near optimum solution efficiently, as discussed in the following section.

Remark. Note that the above normal approximations of $\lambda(x, t), \mu(x, t)$ and $\theta(x, t)$ work well only when $\lambda(x, t)$ and $\mu(x, t)$ have large means. Note that this may not be the case all the time, e.g., when $t \rightarrow 0$ or the trip production and attraction at partition $\mathcal{X}_{\mathbf{x}}(x)$ are low. Note that the above cost component formulations (9) - (12) are integrals of these terms over time and space. In these integrals with a relatively large time span (e.g., up to $\bar{t}$ ), weights from integrands with $t \rightarrow 0$ are negligible. Therefore, if the travel demand is not too small overall, the cost estimations based on the normal approximations should be accurate. However, if the travel demand is sparse such that a large portion of partitions have $\lambda(x, t)$ or $\mu(x, t)$ with relatively small means (e..g, less than 10) for a significant time period, the proposed approximations may have visible errors in estimating the total system cost. Further, when $\gamma\left(x^{\prime}, y^{\prime}, t\right)$ values are dependent across space points $x^{\prime}$ and $y^{\prime}$

and time $t$, these approximations based on the central limit theories may not be valid. However, if the spatial and temporal correlations of these $\gamma\left(x^{\prime}, y^{\prime}, t\right)$ values can be analytically formulated (e.g, with a covariance matrix), this framework still works with minor adaptations incorporating these correlations. While this extension is interesting, it is out of this paper's scope and we leave it for future studies.

\section{Continuum Approximation (CA)}

A CA approach basically approximates each local neighborhood of a space with an infinite homogeneous plane (IHP), where every parameter is homogenous and thus the optimal location design is very easy to solve. If the total cost in this neighborhood is not much affected by other distance areas and all settings in the original space vary relatively slowly, then this IHP solution shall well approximate the optimal location design in this neighborhood of the original space. Finally, integrating the approximated location designs for all neighborhoods shall yield a fairly good approximation of the true optimal location design for this whole space. Note that in our problem, the total cost in a neighborhood only depends on the partition shape and the inventory level around this neighborhood and is not much affected by the distant settings. And we focus on a space where all parameters change relatively smoothly around each neighborhood. These features justify the adoption of a CA method. Note that our CA method has two fundamental differences from Carlsson and Jia (2013) on the continuous hub-and-spoke problem. First, Carlsson and Jia (2013) focuses on spaces with special geographic structures, while our study investigates a general heterogeneous space. Second, out problem is a non-trivial generalization of the basic hub-and-spoke problem that 
incorporates complicated additional components such as dynamic and uncertain demand, nonlinear EV charging time and vehicle fleet operations. This section will first formulate an IHP problem and then discuss how to integrate the IHP solutions into an approximate solution for the original heterogeneous space.

\subsection{Infinite Homogeneous Plane (IHP)}

An IHP is a two-dimensional infinite space ,i.e., $\mathbb{R}^{2}$, where all parameters are constant or homogenous across all locations. Let $\gamma^{+}(x, t)$ denote the randomly generated trip rate per unit area at location $x \in \mathbb{R}^{2}$ and time $t \in[0, \bar{t}]$, which follows a distribution with homogeneous mean $\bar{\gamma}^{+}(t)$ and standard deviation $\hat{\gamma}^{+}(t)$. Let $\gamma^{-}(x, t, s)$ denote the random rate of arriving EVs at the SOC of $s \in[0, \bar{s}]$ per unit-area around location $x \in \mathbb{R}^{2}$ at time $t$, which follows a distribution with homogeneous mean $\bar{\gamma}^{-}(t, s)$ and standard deviation $\hat{\gamma}^{-}(t, s)$. Again we assume both $\gamma^{+}(x, t)$ and $\gamma^{-}(x, t, s)$ are spatially and temporally independent. Building an EV sharing facility anywhere on this IHP costs a fixed construction cost $f$. The built facilities are evenly distributed on $\mathbb{R}^{2}$ and divide $\mathbb{R}^{2}$ into identical regular hexagons with Voronoi tessellations ${ }^{4}$. We use $A$ to denote the area size of such a hexagon covered by a facility. Since all hexagon partitions are transitionally symmetrical, we will only examine one generic hexagon in the following analysis.

Let $\mathcal{A}$ denote the set of locations in this generic hexagonal partition. The cumulative departure count within this partition by time $t \in[0, \bar{t}]$, denoted by $\lambda(t)$, is formulated as $\int_{x \in \mathcal{A}} \int_{0}^{t} \gamma^{+}\left(x, t^{\prime}\right) d t^{\prime}$. Since the distribution of $\gamma^{+}(x, t)$ is spatially and temporally independent and homogeneous across this partition, based on the central limit theory, $\lambda(t)$ shall approximately follow a normal distribution $\mathcal{N}\left(A \bar{\lambda}(t), A \hat{\lambda}(t)^{2}\right)$ where

$$
\bar{\lambda}(t):=\int_{0}^{t} \bar{\gamma}^{+}\left(t^{\prime}\right) d t^{\prime}
$$

and

$$
\hat{\lambda}(t):=\left(\int_{0}^{t} \hat{\gamma}^{+}\left(t^{\prime}\right)^{2} d t^{\prime}\right)^{0.5}
$$

The cumulative arrival count (of available vehicles) in a partition by time $t \in[0, \bar{t}]$, denoted by $\mu(t)$, can be formulated as $\int_{x \in \mathcal{A}} \int_{0}^{t} \int_{0}^{\bar{s}} \gamma^{-}\left(x, s, t^{\prime}-H(s)\right) d s d t^{\prime}$. Again, due to the central limit theory, $\mu(t)$ shall approximately follow normal distribution $\mathcal{N}\left(A \bar{\mu}(t), A \hat{\mu}(t)^{2}\right)$ where

$$
\bar{\mu}(t):=\int_{0}^{t} \int_{0}^{\bar{s}} \bar{\gamma}^{-}\left(s, t^{\prime}-H(s)\right) d s d t^{\prime}
$$

and

$$
\hat{\mu}(t):=\left(\int_{0}^{t} \int_{0}^{\bar{s}} \hat{\gamma}^{-}\left(s, t^{\prime}-H(s)\right)^{2} d s d t^{\prime}\right)^{0.5} .
$$

This yields the cumulative net departure count

$$
\theta(t)=\lambda(t)-\mu(t), \forall t \in[0, \bar{t}]
$$

\footnotetext{
${ }^{4}$ Hexagon tessellations are usually used in CA because they yield less transportation cost than other regular (i.e., triangle and rectangle) tessellations (Toh and Higgins, 1985; Cui et al., 2010).
} 
which approximately follows $\mathcal{N}\left(A \bar{\theta}(t), A \hat{\theta}(t)^{2}\right)$ where

$$
\bar{\theta}(t):=\bar{\lambda}(t)-\bar{\mu}(t)
$$

and

$$
\hat{\theta}(t):=\left(\hat{\lambda}(t)^{2}+\hat{\mu}(t)^{2}\right)^{0.5} .
$$

Note that deviation $\hat{\theta}(t)$ increases with time $t$. Further, parameters $\bar{\lambda}(t), \hat{\lambda}(t), \bar{\mu}(t), \hat{\mu}(t), \bar{\theta}(t)$ and $\hat{\theta}(t)$ are exogenous inputs and independent of partition size $A$.

Now we will formulate the total unit-area cost of this IHP with these homogeneous settings as a function of partition area $A$. Similar to equation (1), the unit-area facility construction cost can be formulated as

$$
C_{f}(A):=f A^{-1} .
$$

Within a hexagonal partition, all generated trips shall walk to the facility at the centroid of this hexagon to pick up EVs. Based on equation (2) and the results from the classic CA method (Daganzo and Newell, 1986), the average walking cost per unit-area in a hexagon partition is

$$
C_{w}(A):=w(\bar{\lambda}(\bar{t})+\bar{\mu}(\bar{t})) \eta g A^{0.5},
$$

where factor $g$ is the distance between the centroid and an average point in a regular hexagon of area size 1 (and thus the average Euclidean distance between an average trip generation point in the partition to the seed facility is $g A^{0.5}$ ). With previous experiments (Li and Ouyang, 2010), $g$ can be fairly well approximated by $\frac{2}{3 \sqrt{\pi}}$.

Again, we can use the FTA or PTA criterion to determine the initial EV inventory that assures service reliability. Without much loss of generality, we allow an EV inventory to be fractional in the IHP analysis ${ }^{5}$. The initial EV inventory at a facility is essentially a function of $A$, which we denote by $I(A)$. Then the unit area inventory holding cost is

$$
C_{h}(A):=h I(A) A^{-1} .
$$

If FTA is applied with the fixed time $\hat{t}$, similar to equation (7) we can derive that $I(A)$ shall be no less than

$$
I^{F}(A):=\left[\bar{\theta}(\hat{t}) A+\Phi^{-1}(\alpha) \hat{\theta}(\hat{t}) A^{0.5}\right]^{+} .
$$

If PTA is applied, similar to equation (8), the minimum $I(A)$ shall be equal to

$$
I^{P}(A):=\max _{t \in[0, \bar{t}]} \bar{\theta}(t) A+\Phi^{-1}(\alpha) \hat{\theta}(t) A^{0.5} .
$$

At the period end time $\bar{t}$, the inventory level of a facility, if lower than $I(A)$, needs to be rebalanced to $I(A)$ by bringing vehicles in nearby facilities that have redundancy. Similar to equation (10), the expected inventory that needs to be balanced at a facility in the IHP is

$$
I_{\bar{t}}(A):=\int_{0}^{\infty} z d \Phi\left(\frac{z-\bar{\theta}(\bar{t}) A}{\hat{\theta}(\bar{t}) A^{0.5}}\right)=A^{0.5} \hat{\theta}(\bar{t}) \phi\left(\frac{\bar{\theta}(\bar{t}) A^{0.5}}{\hat{\theta}(\bar{t})}\right)+A \bar{\theta}(\bar{t}) \Phi\left(\frac{\bar{\theta}(\bar{t}) A^{0.5}}{\hat{\theta}(\bar{t})}\right),
$$

\footnotetext{
${ }^{5}$ Continuity in this paper means absolute continuity, and thus any continuous function mentioned in this paper should be semi-differentiable everywhere.
} 
where $\phi(\cdot)$ is the standard normal probability distribution function $(\mathrm{PDF})$. Apparently, $I_{\bar{t}}(A)$ is continuous. This yields the unit-area vehicle rebalancing cost as

$$
C_{b}(A):=b I_{\bar{t}}(A) A^{-1} .
$$

With the formulations of these cost components, the total unit-area cost in the IHP is

$$
\begin{aligned}
C(A) & :=C_{f}(A)+C_{w}(A)+C_{h}(A)+C_{b}(A) \\
& =f A^{-1}+w(\bar{\lambda}(\bar{t})+\bar{\mu}(\bar{t})) \eta g A^{0.5}+h I(A) A^{-1}+b I_{\bar{t}}(A) A^{-1}
\end{aligned}
$$

Then the IHP design problem becomes finding the optimal partition size $A$ to minimize the unit area cost $C(A)$, i.e.,

$$
C^{*}:=\min _{A \in(0, \infty)} C(A)
$$

Note that the above problem has only one single variable $A$. This uni-variable optimization problem is much simpler that the original system design problem (13). If function $C(A)$ is unimodal, then it can be solved by a bisection search algorithm that has only a sub-linear time complexity. We will investigate properties related to the unimodality of function $C(A)$ in the following analysis. The proofs of the following lemmas and propositions are available in Appendix B.

Lemma 4.1. Function $C(A)$ is continuous, non-negative and $\lim _{A \rightarrow 0^{+}} C(A)=\infty, \lim _{A \rightarrow \infty} C(A)=$ $\infty$.

For a generic continuous function, we use an overhead dot to represent the set of subgradients (e.g., $\dot{C}(A)$ is the set of sub-gradients of $C(A)$ with respect to variable $A$ ). For the convenience of analysis, we made the following generalizations from single value operations to set operations. First, with slight abuse of the notation, we view a single element equivalent as a set including this single element only, which allows us to unify operations between sets and single elements. We generalize the following operators between two sets $X$ and $Y$ :

$$
\begin{gathered}
X<(\leq,>, \geq \text { or }=) Y \text {,iff } x<(\leq,>, \geq \text { or }=) y, \forall x \in X, y \in Y ; \\
X+(-, \times \text { or } /) Y=\{x+(-, \times \text { or } /) y, \forall x \in X, y \in Y\} .
\end{gathered}
$$

Further, we say that a set-valued function, say $\dot{C}(A)$, is increasing (or decreasing ) at $A$, if for an arbitrarily small $\epsilon>0, \dot{C}(A) \leq($ or $\geq) \dot{C}(A+\epsilon)$. The increasing (or decreasing) is strict if this inequality becomes strict.

Lemma 4.2. There exists a finite $A^{*} \in(0, \infty)$ such that $C\left(A^{*}\right)=C^{*}$ and $0 \in \dot{C}\left(A^{*}\right)$.

Since $I(A)$ is continuous, and thus $\dot{I}(A)$ exists $\forall A \in(0, \infty)$. Then we can show that function $C(A)$ is strictly unimodal and has a unique minimum if $\dot{I}(A)$ satisfies the following condition.

Proposition 4.1. Define $G(A):=I(A) A^{-1}$. If $\dot{G}(A) A^{\delta}$ is increasing over $A \in(0, \infty)$ for some $\delta \in[0.5,1.5]$, there exists a $A^{*} \in(0, \infty)$ such that $0 \in \dot{C}\left(A^{*}\right), \dot{C}(A)<0, \forall A \in\left(0, A^{*}\right)$ and $\dot{C}(A)>$ $0, \forall A \in\left(A^{*}, \infty\right)$; i.e., function $C(A)$ strictly unimodal with a unique minimizer of $A^{*} \in(0, \infty)$. 
Proposition 4.2. If $I(A)=I^{F}(A)$ (i.e., when the FTA criterion is adopted), $C(A)$ is strictly unimodal with a unique minimizer over $A \in(0, \infty)$.

Proposition 4.3. If $I(A)=I^{P}(A)$ (i.e., when the PTA criterion is adopted), $C(A)$ is strictly unimodal with a unique minimizer over $A \in(0, \infty)$.

For strictly unimodal $C(A)$, we can find the optimal area size $A^{*}$ with the following bisection algorithm in an efficient sub-linear time.

B1: Set $\underline{A}=\bar{A}=1$. Set an error tolerance $\epsilon$.

B2: While $\dot{C}(\underline{A}-)$ (the left differential of $C(\underline{A})$ ) is positive, $\underline{A}=\underline{A} / 2$. While $\dot{C}(\bar{A}+$ ) (the right differential of $C(\bar{A}))$ is negative, $\bar{A}=2 \bar{A}$.

B3: Set $A=(\underline{A}+\bar{A}) / 2$.

B4: If $\dot{C}(A-)>0$, set $\bar{A}=A$; otherwise, set $\underline{A}=A$.

B5: If $\bar{A}-\underline{A} \geq \epsilon$, go to B4; otherwise, return $A^{*}=A$.

\subsection{Continuum Approximation (CA) for a Heterogeneous Space}

\subsubsection{Numerical Model}

This section discusses how we can apply the IHP results to the original finite heterogeneous space $\mathcal{X}$. We assume that functions $f(x), \bar{\gamma}(x, y, t)$ vary relatively slowly over $x \in \mathcal{X}$. Instead of looking for discrete locations $\mathbf{x}$ directly, we propose to look for a continuous function, $A(x) \in \mathbb{R}^{+}, x \in \mathcal{X}$, that approximates the partition area size of a facility near $x$; i.e., $A(x) \approx\left|\mathcal{X}_{\mathbf{x}}\left(S_{\mathbf{x}}(x)\right)\right|$. Further, since we assume that the EV sharing facilities are densely located, the size of $\mathcal{X}$ should be much larger than an $A(x)$, i.e., $|\mathcal{X}| \gg A(x), \forall x \in \mathcal{X}$, and thus the heterogeneity around the boundary of $\mathcal{X}$ can be ignored without affecting much the total cost. When all settings are approximately constant over a region comparable to the size of several initial service areas, $A(x)$ should also be approximately constant on that scale as well, and the CA result should be very close to the true optimum. Even if moderate heterogeneity is present on this scale, since the unit-area cost function (17) has an similar structure to an EOQ (economic order quantity) function that has an optimal solution insensitive to parameter variations (Daganzo, 2005), the CA solution should be an good approximation of the true optimum as well.

We apply the cost formulation (17) to each neighborhood of $x \in \mathcal{X}$ (i.e., imagining that this neighborhood is part of an IHP). Given the parameters of the original plane $\mathcal{X}$, the IHP parameters for a neighborhood around $x$ can be constructed in the following way. We set

$$
\begin{gathered}
F=f(x), \\
\bar{\gamma}^{+}(t)=\int_{y \in \mathcal{X}} \bar{\gamma}(x, y, t) d y, \\
\hat{\gamma}^{+}(t)=\left(\int_{y \in \mathcal{X}} \hat{\gamma}^{2}(x, y, t) d y\right)^{0.5}, \\
\bar{\gamma}^{-}(s, t)=\int_{y \in \mathcal{X} \text { s.t. } D(x, y)=\bar{s}-s} \bar{\gamma}(y, x, t-\bar{s}+s-H(s)) d y,
\end{gathered}
$$




$$
\hat{\gamma}^{-}(s, t)=\left(\int_{y \in \mathcal{X} \text { s.t. } D(x, y)=\bar{s}-s} \hat{\gamma}^{2}(y, x, t-\bar{s}+s-H(s))\right)^{0.5} d y .
$$

We plug these input parameters into the IHP analysis approach described in Section 4.1, and we can efficiently solve the optimal partition area to problem (18), denoted by $A^{*}(x)$, for each neighborhood $x$. After we solve $A^{*}(x)$ for all neighborhoods $x \in \mathcal{X}$, we can accordingly derive the optimal vehicle inventory at each neighborhood $x$, denoted by $I^{*}(x):=I\left(A^{*}(x)\right)$, where function $I(A)$ is defined by equation (14) if the FCA criterion is adopted or by equation (15) if PVA is adopted. Then the optimal objective to the original problem (13) can be estimated by

$$
\bar{C}^{*}=\int_{x \in \mathcal{X}} C\left(A^{*}(x)\right) d x .
$$

Since the inverse of $A^{*}(x)$ denote the optimal facility density at $x$, the total number of optimal car sharing stations can be estimated by

$$
N^{*}:=\int_{x \in \mathcal{X}}\left(A^{*}(x)\right)^{-1} d x
$$

\subsubsection{Discretization Issues}

Continuous solution $A^{*}(x)$ may not be able to directly applied for discrete charging facility installations. A discretization approach is needed to convert $A^{*}(x)$ to discrete location design $\mathbf{x}$. Ouyang (2006) proposed a novel meta-heuristic disk model that can solve discrete location design with the CA output in a relatively efficient manner. The disk model exerts repulsive forces to $N^{*}$ disks that each represents the initial partition of a facility, and iteratively adjusts positions and sizes of these disks to achieve optimal layout. However, this algorithm needs a careful selection of model parameters to assure the convergence of the solution, and it requires relatively large computational time and space for extremely large instances that we may test. Thus we opt to adopt a construct-heuristic algorithm proposed by Li (2015) to obtain a discrete solution. This algorithm first divides space $\mathcal{X}$ into small square cells and sets the origin at a corner of space $\mathcal{X}$. The cell size should be less than the minimum service area, i.e., $\min _{x \in \mathcal{X}} A^{*}(x)$. Then starting from the origin cell, it iteratively calls a construction subroutine to partition these cells into service areas. This subroutine grows the current service area from the starting cell by visiting nearby cells in a way that the visited cells form a nearly regular shape (e.g., square). This subroutine stops when the integral of facility density $A^{*-1}(x)$ in the current service area is exactly identical to 1 (a cell may be split to a fraction to make sure the equality is exact). Then this algorithm starts a new subroutine to construct the next service area from the first unvisited cell (ranked first along the clockwise ring direction and then along the radial direction starting from the origin). This subroutine will be repeated until all cells in $\mathcal{X}$ are visited. Then space $\mathcal{X}$ is partitioned into a number of service areas that are mostly close to a regular shape, and the density centers of these service areas are the discrete facility locations. This method requires only a linear computational time and space (i.e., by sweeping the space only once), and the service area covered by each facility in this solution is very consistent with the continuous function $A^{*}(x)$ (i.e., the integral of $A^{*-1}(x)$ in all service areas except the last one is identical to 1). Once the discrete location $\mathbf{x}$ is obtained, we can plug it into model (13) to obtain the total cost with respect to this discrete design. 
Since it is difficult to analytically solve the above integral equations, especially for large-scale problem instances, the following simple numerical integration method can be used to approximate integrals of spatial and temporal functions in a discrete manner. We basically partition space $\mathcal{X}$ into a set of equal-area unit squares $\mathcal{Q}$, each unit square $q \in \mathcal{Q}$ with a central coordinate of $x_{q}$ and a identical width of $\Delta l$ (and thus the area of $q$ is $\Delta l^{2}$ ), then a spatial integration with respect to a generic function $F(x), \forall x \in \mathcal{X}$ can be approximated by

$$
\int_{x \in \mathcal{X}} F(x) d x \approx \sum_{q \in \mathcal{Q}} F\left(x_{q}\right) \Delta l^{2} .
$$

Similarly, we can discretize the continuous time period $[0, \bar{t}]$ into discrete time points separated by a small interval $\Delta t$, and with respect to a generic function $F(t), \forall t \in[0, \bar{t}]$, a temporal integral can be approximated by

$$
\int_{t=0}^{\bar{t}} F(t) d t \approx \sum_{i=0}^{\bar{t} / \Delta t} F(i \Delta t) \Delta t,
$$

and a maximum can be approximated by

$$
\max _{t \in[0, \bar{t}]} f(t) \approx \max _{i=0,1, \cdots, \bar{t} / \Delta t} f(i \Delta t) .
$$

The size of $\Delta l$ and $\Delta t$ can be adjusted to balance computational time and approximation accuracy.

\section{$5 \quad$ Numerical Examples}

This section illustrates the proposed CA model with several numerical examples. All experiments were performed on a $\mathrm{PC}$ with a quad-core $2.97 \mathrm{GHz} \mathrm{CPU}$ and a $16 \mathrm{~GB}$ RAM. The default parameter values are set as follows. The operational cycle $\bar{t}$ is set to be 24 hours, which is a natural operational period. In numerical calculations, the 24 hour period is discretized to evenly distributed time points separated by $\Delta t=5$ minutes, which yields 288 time intervals. The daily prorated fixed cost is assumed to $f=\$ 500$ including prorated construction cost and daily fixed operation cost (e.g., labor and facility maintenance). The unit distance walking cost $w$ is set to be $\$ 6.25$ per kilometer (based on a walking speed of $4 \mathrm{~km} / \mathrm{h}$ (Tirachini et al., 2014) and a value of time of $\$ 25$ per hour (Tirachini et al., 2014)). The life cycle cost of an EV is set to be $\$ 83,000$, which is the average life of cycle cost of several models of Ford Taurus with electric batteries investigated in Delucchi and Lipman (2001). Assuming that a vehicle has a service life of 5 years, then the daily vehicle holding cost $h=\$ 45.5$ per day. The vehicle handling cost $b$ in rebalancing vehicle inventories at the end of day is assumed to be $\$ 20$ per vehicle. We assume that the vehicle's average speed is $v=40 \mathrm{~km} / \mathrm{h}$. We set the travel range $\bar{s}$ of an electrical vehicle of $160 \mathrm{~km}$, a full-cycle charging time $\bar{r}$ of 7 hours (based on the characteristics of Nissan Leaf and a level-2 charging station reported by Yilmaz and Krein (2012)). Based on the experiments from Chen (2007), we assume that in a full charging cycle, it takes $\frac{\bar{r}}{12}$ time to charge the first $40 \%$ of $\bar{s}$ and $\frac{11 \bar{r}}{12}$ time to charge the remaining $60 \%$ of $\bar{s}$, which implies it takes a relatively long time to charge when an vehicle's remaining SOC is relatively high. Then the corresponding charging function is

$$
H(s)=\min \left\{\frac{s}{0.6 \bar{s}}, 1\right\} \frac{11 \bar{r}}{12}+\max \left\{\frac{s-0.6 \bar{s}}{0.4 \bar{s}}, 0\right\} \frac{\bar{r}}{12} .
$$


Table 1: Parameter default values.

\begin{tabular}{c|c}
\hline Parameter & Default Value \\
\hline$f$ & $\$ 500 /$ station \\
$w$ & $\$ 6.25 / \mathrm{km}$ \\
$h$ & $\$ 45.5 / \mathrm{EV}$ \\
$b$ & $\$ 20 / \mathrm{EV}$ \\
$v$ & $40 \mathrm{~km} /$ hour \\
$\bar{s}$ & $160 \mathrm{~km}$ \\
$\bar{r}$ & 7 hour \\
\hline
\end{tabular}

The default values of these key parameters are summarized in Table (1).

\subsection{Sioux-Falls Network}

This case study uses the hourly OD trip data between the 24 nodes of the transportation network of the Sioux-Falls city, North Dakota, US, available through the website "Transportation Network Test Problems", prepared by Dr. Bar-Gera (http://www.bgu.ac.il/ bargera/tntp/). The total number of trips are 173,100. The actual node coordinates are obtained by manually querying the adjusted node locations (Chakirov and Fourie, 2013) in the Google Maps (https:// www.google.com/maps). These coordinates are converted to the Universal Transverse Mercator (UTM) coordinate system (Zone 14) using the "PyProj" package of the Python script language. With these original data, we create the input to the CA model as follows. The border points of space $\mathcal{X}$ is created by expanding the convex hull of the 24 nodes along the radial direction from the centroid of the convex hull such that each corner point is $20 \%$ more distance from the centroid. The space is diced into unit squares of width $\Delta l=0.1 \mathrm{~km}$. The OD trips between the original 24 nodes are interpolated across the whole space and then smoothed by the moving average method with a radius of $1 \mathrm{~km}$. We also remove the trips within a travel distance less than $\underline{d}=1 \mathrm{~km}$ that we assume will walk to their destinations. The distributions of daily trip productions, attractions and their difference (i.e. net productions) are plotted in Figure 2. We see from this figure that both productions and attractions vary drastically over the space. We measured that the relative deviation (the ratio of the standard deviation to the mean) of the productions is $118 \%$ and the relative deviation of the attractions is $80 \%$.

The trips are prorated to a day with the time-of-day factors shown in Figure 3 (the aggregated time-of-day factors of all types of trips provided by Kansas Department of Transportation (Kansas Department 2009)). We can see in Figure 3 that there are dramatic variations of these factors across 24 hours. the relative deviation of these factors are $77 \%$. This yields the mean values of unit area OD demand $\bar{\gamma}(x, y, t)$. We assume that trip generation between any two locations is a time-varying Poisson process, and thus $\hat{\gamma}(x, y, t)=\bar{\gamma}(x, y, t)^{0.5}$.

We will first examine the performance of the CA approach with the following experiments. Tables 2 and 3 show the relative errors of the cost components between the CA approach and the discretization algorithm and their solution times under the FTA $(\hat{t}=8 \mathrm{am})$ and PTA criteria, respectively. The problem instances are derived by varying the default parameter values set in Table 1 one at a time. The "benchmark" instance uses the default parameter values, and each of other instances varies only one default parameter value as indicated in columns "parameter" and "value". We see that the relative errors of the total cost $\bar{C}$ are all under $9 \%$ and all but 

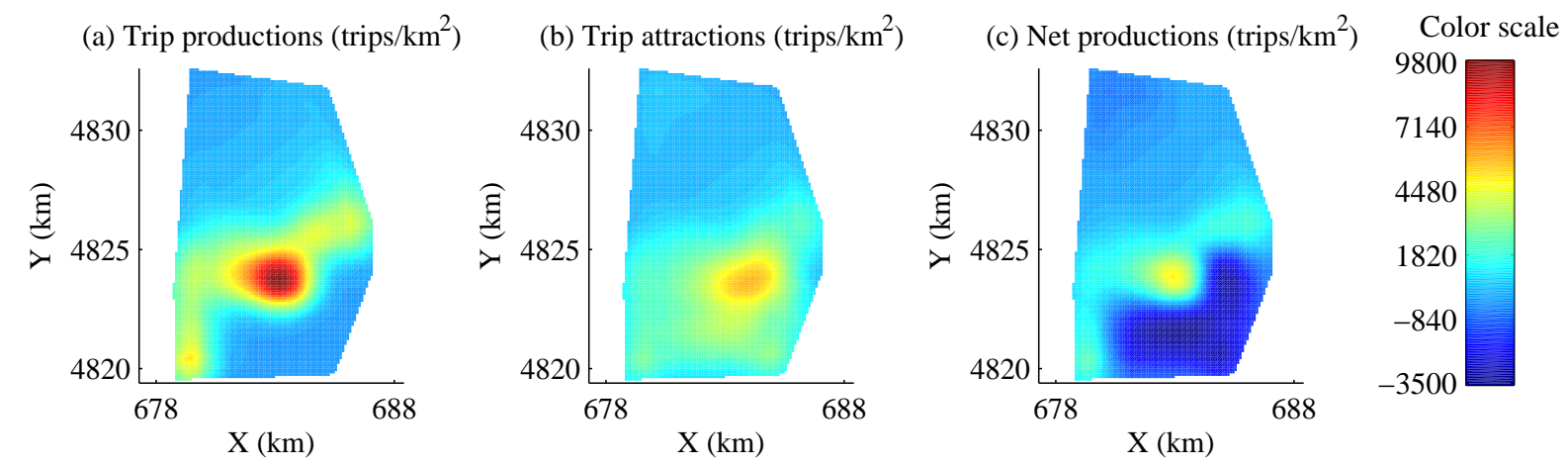

Figure 2: Distributions of (a) trip productions, (b) trip attractions and (c) net trip productions (productions minus attractions) for the Sioux-Falls case.

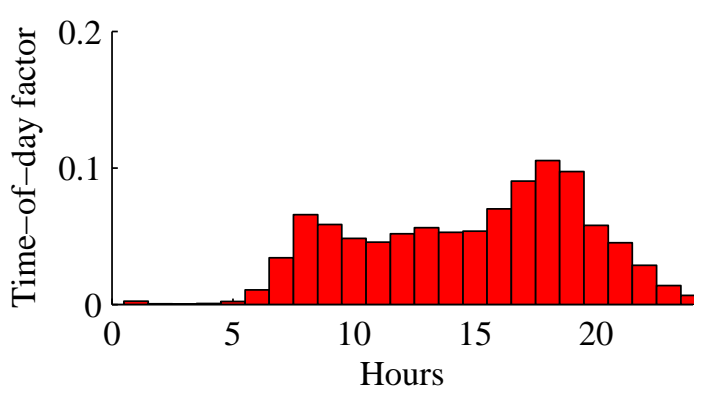

Figure 3: Time-of-day factors. 
two instances are under $6 \%$. The average relative error of $\bar{C}$ is $2.9 \%$ and $4.9 \%$, respectively for the FTA and PTA criteria. These results indicate that the CA approach estimates the total cost of the corresponding discrete solution with a good accuracy, which shall satisfy requirements of most engineering applications. Further, note that these errors are much smaller than the relevant deviation values of several major input parameters, e.g, $118 \%$ for trip productions, $80 \%$ for trip attractions and $77 \%$ for the time-of-the day factors. This shows that the proposed CA approach performs well even when parameter variations are drastic, and we anticipate the relative errors will further reduce as the parameter variations further drop (which is likely the case for cities bigger than Sioux-Falls). Among the four cost components, the errors of fixed facility cost $\bar{C}_{f}$ is negligible $(<0.3 \%)$. The errors of $\bar{C}_{w}$ are all within $2.8 \%$, and most of them are negative, implying that CA tends to slightly underestimate the total walking cost. This is probably because of the minor difference between the actual partition shapes in the discrete solution and the ideal regular hexagon assumed in the CA approach. The errors of $\bar{C}_{h}$ and $\bar{C}_{b}$ are dominating the the total error of $\bar{C}$. These errors are all positive, meaning that CA tend to overestimate EV holding and rebalancing cost. This is probably because that when the generated trips are not perfectly homogeneous over space, the aggregated trip rate in a discrete partition has a relatively low standard deviation compared to the homogeneous case, and this would reduce the needed EVs to maintain the same service level. Nevertheless, the conservative estimates of the EV inventory from CA can help further improve the service reliability and thus shall be acceptable. Comparing the $\bar{C}_{h}$ and $\bar{C}_{b}$ errors (their absolute values) from the two criteria, we see that those for PTA are in general a little greater than those for FTA. This is because in addition to the spatial aggregation, the EV inventory in a discrete partition under the PTA criterion is also a result of temporal aggregation. Due to the variation of the time-of-day factors, this temporally aggregated inventory could be even lower. In addition, we also measured the relative difference of $\bar{C}_{d}$ between the location-design-independent CA solution (12) and the discrete solution, although we do not consider it in the optimal system design. We see that they are all less than $1.2 \%$ and mos of them are less than $0.5 \%$. This confirms the earlier conjecture that the driving cost is not much affected by the system design and can be safely removed from the optimization objective (13).

From Tables 2 and 3, we also see that all CA instances can be solved very efficiently, i.e., within 10 seconds. And the corresponding discretizaiton times are all less than 1000 seconds. This implies that the cost for planning a complex EV sharing system can be approximately estimated by the CA approach very efficiently. Further, a near-optimum discrete design can be obtained in a reasonable time, whereas a pure discrete model dealing with 173,100 individual trips and 288 time intervals could be easily computationally intractable.

In the following experiments, we use the PTA criterion since it holds a better service reliability compared to FTA. Figures 4 and 5 plot how cost components $\bar{C}_{f}, \bar{C}_{w}, \bar{C}_{h}$ and $\bar{C}_{b}$ (obtained from the CA approach) and relevant measures vary to the increases of the key parameters. To quantify sensitivity, we define a sensitivity factor for each cost component-parameter pair as the ratio of the cost component value when the corresponding parameter is 2 times its benchmark value to that when this parameter is 0.2 times its benchmark value. We annotate each sensitivity factor value on the corresponding cost component-parameter curve in Figures 4 and 5 .

Figure 4 shows how the cost components vary with the corresponding cost rates. Figures 4 (a), (b) and (c) examine sensitivity to fixed station cost $f$. We see that in Figure 4 (a), as $f$ increases, the total fixed cost $\bar{C}_{f}$ and the total walking cost $\bar{C}_{w}$ both increase while the increasing slopes gradually flatten. The corresponding sensitivity factors indicate that as $f$ increases from $\$ 100 /$ sta- 
Table 2: Relative cost errors and solution times under the FTA $(\hat{t}=8$ am) criterion for the Sioux-Falls network.

\begin{tabular}{|c|c|c|c|c|c|c|c|c|c|}
\hline \multirow{2}{*}{ Parameter } & \multirow{2}{*}{ Value } & \multicolumn{6}{|c|}{ Relative errors } & \multicolumn{2}{|c|}{ Solution times (secs) } \\
\hline & & $\bar{C}$ & $\bar{C}_{f}$ & $\bar{C}_{w}$ & $\bar{C}_{h}$ & $\bar{C}_{b}$ & $\bar{C}_{d}$ & $\mathrm{CA}$ & Discretization \\
\hline \multicolumn{2}{|c|}{ Benchmark } & $2.7 \%$ & $0.0 \%$ & $-2.3 \%$ & $3.7 \%$ & $5.0 \%$ & $0.3 \%$ & 7 & 799 \\
\hline \multirow{2}{*}{$f$} & $\$ 100$ & $2.3 \%$ & $0.0 \%$ & $-1.6 \%$ & $2.1 \%$ & $3.8 \%$ & $0.1 \%$ & 9 & 793 \\
\hline & $\$ 2500$ & $3.5 \%$ & $0.2 \%$ & $-2.3 \%$ & $5.6 \%$ & $7.9 \%$ & $0.7 \%$ & 9 & 788 \\
\hline \multirow[b]{2}{*}{$w$} & $\$ 3.125 / \mathrm{km}$ & $5.0 \%$ & $0.0 \%$ & $-1.6 \%$ & $4.9 \%$ & $7.6 \%$ & $0.3 \%$ & 8 & 788 \\
\hline & $\$ 12.5 / \mathrm{km}$ & $1.1 \%$ & $0.1 \%$ & $-2.3 \%$ & $2.5 \%$ & $3.0 \%$ & $0.1 \%$ & 9 & 789 \\
\hline \multirow{2}{*}{$h$} & $\$ 9.1 /$ veh & $2.4 \%$ & $-0.1 \%$ & $-2.3 \%$ & $3.4 \%$ & $4.8 \%$ & $0.2 \%$ & 8 & 788 \\
\hline & $\$ 227.4$ /veh & $3.8 \%$ & $-0.3 \%$ & $-0.8 \%$ & $4.4 \%$ & $6.1 \%$ & $0.5 \%$ & 8 & 793 \\
\hline \multirow{2}{*}{$b$} & $\$ 10 /$ veh & $2.3 \%$ & $0.1 \%$ & $-2.0 \%$ & $3.9 \%$ & $5.3 \%$ & $0.2 \%$ & 8 & 787 \\
\hline & $\$ 40 /$ veh & $3.6 \%$ & $0.1 \%$ & $-2.3 \%$ & $3.5 \%$ & $5.3 \%$ & $0.2 \%$ & 8 & 787 \\
\hline \multirow{2}{*}{$v$} & $20 \mathrm{~km} / \mathrm{h}$ & $3.0 \%$ & $-0.1 \%$ & $-2.1 \%$ & $4.0 \%$ & $5.4 \%$ & $0.3 \%$ & 7 & 802 \\
\hline & $60 \mathrm{~km} / \mathrm{h}$ & $2.6 \%$ & $-0.1 \%$ & $-2.2 \%$ & $3.4 \%$ & $4.9 \%$ & $0.2 \%$ & 7 & 797 \\
\hline \multirow{2}{*}{$\bar{s}$} & $80 \mathrm{~km}$ & $3.2 \%$ & $0.1 \%$ & $-1.9 \%$ & $4.4 \%$ & $5.5 \%$ & $0.2 \%$ & 7 & 803 \\
\hline & $320 \mathrm{~km}$ & $2.2 \%$ & $0.1 \%$ & $-1.9 \%$ & $2.5 \%$ & $4.3 \%$ & $0.3 \%$ & 7 & 791 \\
\hline \multirow{2}{*}{$\bar{r}$} & $1 \mathrm{~h}$ & $1.8 \%$ & $-0.1 \%$ & $-1.9 \%$ & $1.7 \%$ & $3.9 \%$ & $0.2 \%$ & 7 & 788 \\
\hline & $20 \mathrm{~h}$ & $3.1 \%$ & $0.0 \%$ & $-1.9 \%$ & $4.4 \%$ & $5.2 \%$ & $0.2 \%$ & 7 & 804 \\
\hline \multicolumn{2}{|c|}{ Mean (of absolute values) } & $2.9 \%$ & $0.1 \%$ & $2.0 \%$ & $3.6 \%$ & $5.2 \%$ & $0.3 \%$ & 8 & 793 \\
\hline
\end{tabular}

Table 3: Relative cost errors and solution times under the PTA criterion for the Sioux-Falls network

\begin{tabular}{|c|c|c|c|c|c|c|c|c|c|}
\hline \multirow{2}{*}{ Parameter } & \multirow{2}{*}{ Value } & \multicolumn{6}{|c|}{ Relative errors } & \multicolumn{2}{|c|}{ Solution times (secs) } \\
\hline & & $\bar{C}$ & $\bar{C}_{f}$ & $\bar{C}_{w}$ & $\bar{C}_{h}$ & $\bar{C}_{b}$ & $\bar{C}_{d}$ & $\mathrm{CA}$ & Discretization \\
\hline \multicolumn{2}{|c|}{ Benchmark } & $5.2 \%$ & 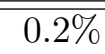 & $-1.7 \%$ & $6.0 \%$ & $7.7 \%$ & $\overline{0.4 \%}$ & 8 & 804 \\
\hline \multirow{2}{*}{$f$} & $\$ 100$ & $3.6 \%$ & $0.1 \%$ & $-0.6 \%$ & $3.8 \%$ & $5.5 \%$ & $0.2 \%$ & 10 & 876 \\
\hline & $\$ 2500$ & $5.9 \%$ & $-0.3 \%$ & $-2.8 \%$ & $7.9 \%$ & $9.6 \%$ & $0.7 \%$ & 9 & 833 \\
\hline \multirow{2}{*}{$w$} & $\$ 3.125 / \mathrm{km}$ & $5.5 \%$ & $0.2 \%$ & $-1.2 \%$ & $6.0 \%$ & $7.2 \%$ & $0.6 \%$ & 9 & 852 \\
\hline & $\$ 12.5 / \mathrm{km}$ & $2.6 \%$ & $-0.1 \%$ & $-2.1 \%$ & $3.5 \%$ & $4.7 \%$ & $0.2 \%$ & 10 & 880 \\
\hline \multirow{2}{*}{$h$} & $\$ 9.1 /$ veh & $3.4 \%$ & $0.0 \%$ & $-2.4 \%$ & $4.7 \%$ & $5.9 \%$ & $0.3 \%$ & 10 & 908 \\
\hline & $\$ 227.4$ /veh & $8.9 \%$ & $-0.3 \%$ & $2.2 \%$ & $9.2 \%$ & $11.6 \%$ & $1.2 \%$ & 10 & 927 \\
\hline \multirow{2}{*}{$b$} & $\$ 10 /$ veh & $4.0 \%$ & $-0.2 \%$ & $-2.1 \%$ & $5.1 \%$ & $6.5 \%$ & $0.4 \%$ & 10 & 883 \\
\hline & $\$ 40 /$ veh & $4.6 \%$ & $0.1 \%$ & $-1.5 \%$ & $4.9 \%$ & $6.2 \%$ & $0.4 \%$ & 10 & 875 \\
\hline \multirow{2}{*}{$v$} & $20 \mathrm{~km} / \mathrm{h}$ & $4.9 \%$ & $0.0 \%$ & $-1.6 \%$ & $5.7 \%$ & $7.1 \%$ & $0.4 \%$ & 8 & 805 \\
\hline & $60 \mathrm{~km} / \mathrm{h}$ & $4.5 \%$ & $-0.1 \%$ & $-2.1 \%$ & $5.2 \%$ & $6.8 \%$ & $0.4 \%$ & 8 & 808 \\
\hline \multirow{2}{*}{$\bar{s}$} & $80 \mathrm{~km}$ & $5.3 \%$ & $-0.2 \%$ & $-1.5 \%$ & $6.1 \%$ & $7.7 \%$ & $0.3 \%$ & 8 & 809 \\
\hline & $320 \mathrm{~km}$ & $5.1 \%$ & $0.1 \%$ & $-1.6 \%$ & $5.8 \%$ & $7.6 \%$ & $0.3 \%$ & 8 & 865 \\
\hline \multirow{2}{*}{$\bar{r}$} & $1 \mathrm{~h}$ & $4.9 \%$ & $-0.1 \%$ & $-1.8 \%$ & $5.6 \%$ & $7.4 \%$ & $0.4 \%$ & 8 & 848 \\
\hline & $20 \mathrm{~h}$ & $5.6 \%$ & $-0.2 \%$ & $-1.4 \%$ & $6.5 \%$ & $7.8 \%$ & $0.4 \%$ & 8 & 822 \\
\hline \multicolumn{2}{|c|}{ Mean (of absolute values) } & $4.9 \%$ & $0.2 \%$ & $1.8 \%$ & $5.7 \%$ & $7.3 \%$ & $0.4 \%$ & 9 & 853 \\
\hline
\end{tabular}



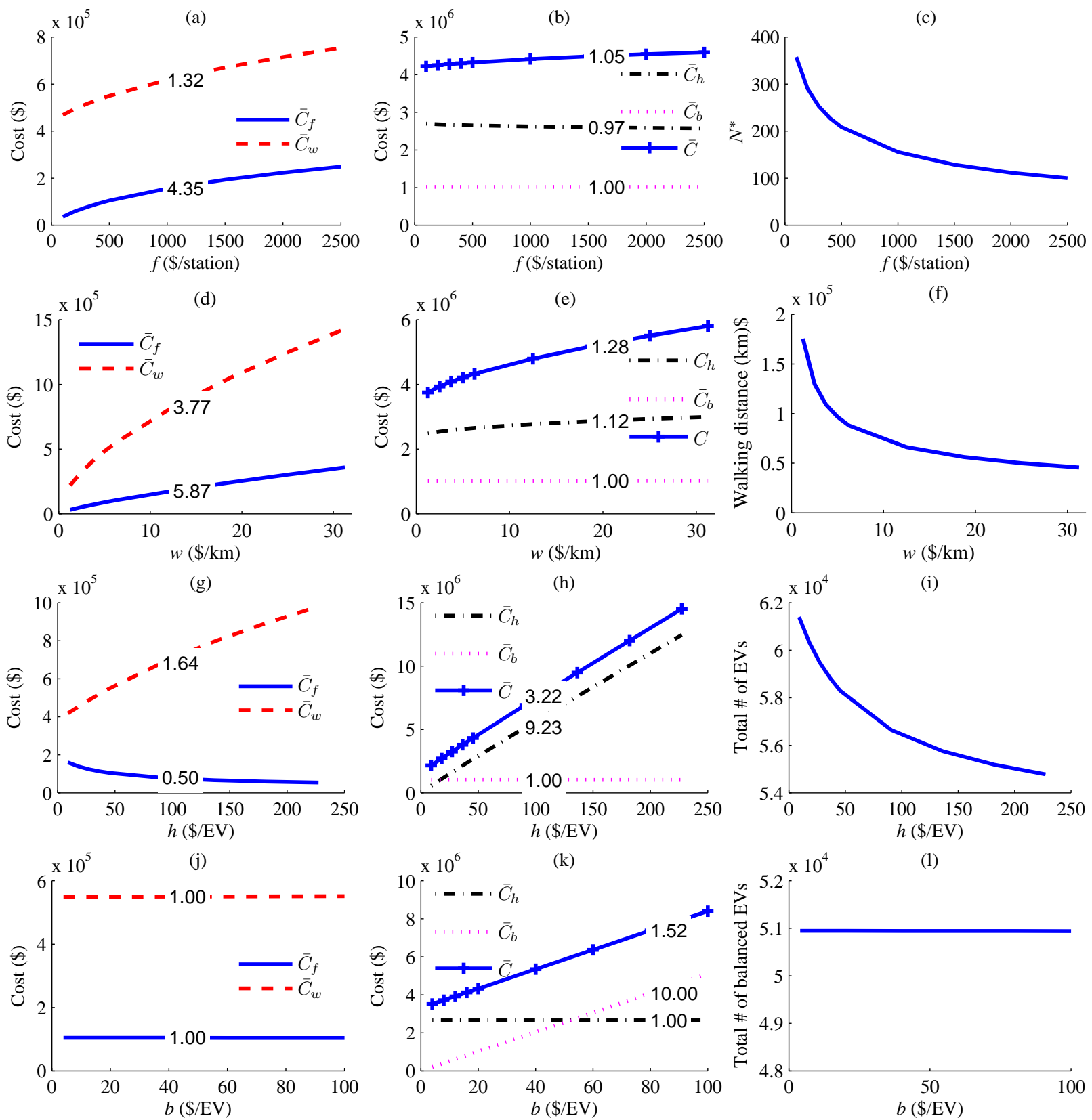

Figure 4: Sensitivity to cost rates $f$ (sub-figures (a) - (c)), $w$ (sub-figures (d) - (f)), $h$ (sub-figures (g) - (i)) and $b$ (sub-figures (j) - (l)). 
tion (or 0.2 time its benchmark value $\$ 500 /$ station) to $\$ 1000 /$ station (or 2 time its benchmark value $\$ 500 /$ station), $\bar{C}_{f}$ increases 4.35 times and $\bar{C}_{w}$ increases 1.32 times. Figure 4 (c) shows that the total number of stations $N^{*}$ decreases from around 360 to around 100 as $f$ increases, which explains the drop of the increasing rate for $\bar{C}_{f}$. Note that the decreasing rate of $N^{*}$ gets smaller as $f$ increases, this implies the decrease of station density gradually slows down as $f$ increases, and this explains why $\bar{C}_{w}$ gradually flattens out as $f$ increases. Figure 4 (b) shows that the fleet cost $\bar{C}_{h}$ slightly decreases with $f$ with a sensitivity factor of 0.97 , the total cost $\bar{C}$ slightly increases with $f$ with a sensitivity factor of 1.05 , and the total rebalancing cost $\bar{C}_{b}$ seems almost independent of $f$ with a sensitivity factor of 1.00 . The slight decrease of $\bar{C}_{h}$ is probably due to the pooling effect from the decrease of the number of stations; i.e., a higher level of aggregation of interdependent stochastic variables can help reduce the relative deviation. Comparing their magnitudes, we see that $\bar{C}_{h}$ is dominating and $\bar{C}_{f}$ is the minimum across all cost components. The decrease of $\bar{C}_{h}$ cancels part of the increase of $\bar{C}_{f}$ and $\bar{C}_{w}$, and thus the increase of $\bar{C}$ is mild.

Figures 4 (d), (e) and (f) show the sensitivity of the optimal results to walking cost rate $w$. Figure $4(\mathrm{~d})$ shows that $\bar{C}_{f}$ increases with $w$ (and so does $N^{*}$ ) with a sensitivity factor of 5.87 . This indicates that as $w$ increases, the station investment (or the station density) shall be significantly raised to slow down the increase of $\bar{C}_{w}$. As a result, the total walking distance decreases significantly as shown in Figure 4 (f). However, due to the increase of the walking cost rate $w$, Figure 4 (d) shows that $\bar{C}_{w}$ increases with a sensitivity factor of 3.77 despite the slowing down trend of the increase rate. Figure 4 (e) shows that $\bar{C}_{h}$ increases slightly with a sensitivity factor of 1.12 , probably because the increase of stations reduces the pooling effect. Again, $\bar{C}_{b}$ is not sensitive to $w$ with a sensitivity factor of 1.00. Overall, the total cost $\bar{C}$ increases with $w$ with a sensitivity factor of 1.28.

Figures $4(\mathrm{~g}),(\mathrm{h})$ and (i) plot the sensitivity results for EV holding cost rate $h$. Figure $4(\mathrm{~g})$ shows that as $h$ increases, $\bar{C}_{w}$ increases (and so does $N^{*}$ ) with a sensitivity factor of 1.64 , but $\bar{C}_{f}$ decreases with a sensitivity factor 0.50 (as a result of increased stations). From Figure 4 (h), we see that $\bar{C}_{b}$ is again insensitive to $h$, and $\bar{C}_{h}$ sharply increases with $h$ with a sensitivity factor of 9.23. Note that in Figure 4 (i), however, the total number of vehicles drops as $h$ increases, probably because the decrease of $N^{*}$ intensifies the pooling effect and thus not as many EVs are needed. Overall the total cost increases significantly with $h$ with a sensitivity factor of 3.22.

Figures $4(\mathrm{j}),(\mathrm{k})$ and (l) plot the sensitivity results for EV balancing cost rate $b$. We see that $\bar{C}_{f}, \bar{C}_{w}$ and $\bar{C}_{h}$ all have a sensitivity factor of 1 and thus none of them is sensitive to $b$. The number of balanced vehicles stays about the same (around 51000) regardless of $b$ value, and thus $\bar{C}_{b}$ increases almost linearly with $b$, which absolutely dominates the growth of $\bar{C}$.

Overall, we see that these each cost component is very sensitive to the corresponding cost rate, whereas other cost components behave in a way that absorbs the impact from the change of this cost rate. Interestingly, we see that $\bar{C}_{b}$ is insensitive to all other cost rates (except $b$ ), and therefore shall not be much affected by the location design. After examining the results, we found that at most facilities, the vehicle rebalancing cost is almost fully determined by the expected count of the net departures and has little to do with its standard deviation. Therefore, aggregating facilities essentially sums the expected values of the corresponding rebalanced EV counts, which does not have much pooling effect and little affects $\bar{C}_{b}$ on a relatively slow-varying space. This implies that removing the EV balance cost from objective (13) would probably not much affect the optimal location and fleet design.

Figure 5 shows how the cost components vary with other relevant variables. Figures 5 (a) and (b) show that all cost components are insensitive to EV speed $v$. This implies that the system design 

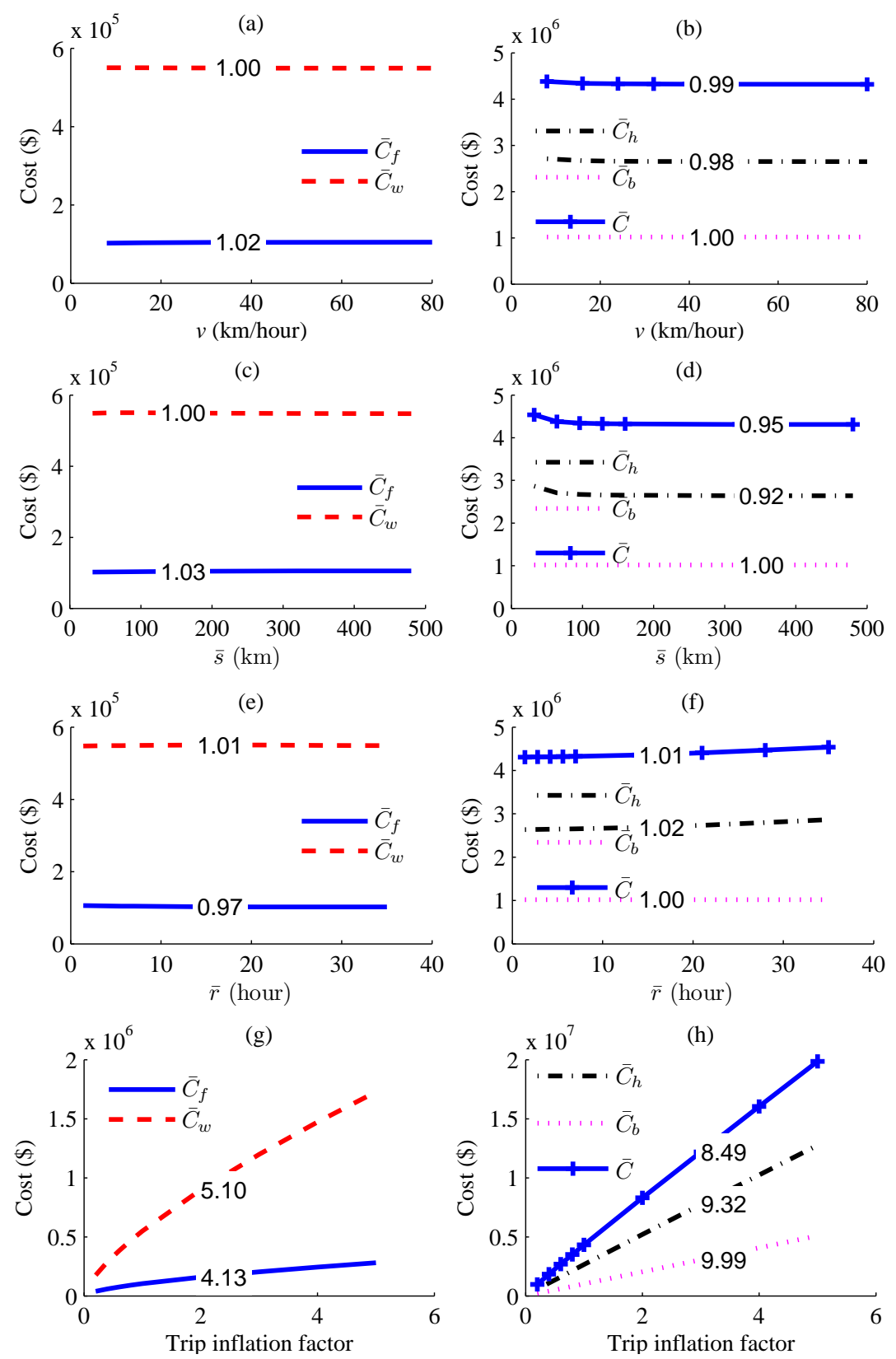

Figure 5: Sensitivity of cost components to cost parameters $v$ (sub-figures (a) \& (b)), $\bar{s}$ (sub-figures (d) \& (d)), $\bar{r}$ (sub-figures (e) \& (f)) and the trip inflation factor (sub-figures (g) \& (h)). 
obtained from CA shall be nearly optimal regardless of the level of traffic congestion. Figures 5 (c) and (d) show that as the maximum SOC $\bar{s}$ increases, $\bar{C}_{f}, \bar{C}_{w}$ and $\bar{C}_{b}$ do not change much, and $\bar{C}_{h}$ and $\bar{C}$ decrease slightly, with sensitivity factors of 0.92 and 0.95 , respectively. This suggests that the increase of the SOC capacity only slightly decreases the total EV fleet size. Figures 5 (e) and (f) show that all cost components are insensitive to the increase of maximum charging time $\bar{r}$. This implies that a long charging time would not much compromise the system performance. In addition, we also inflate input OD trips by an inflation factor. If this trip inflation factor is less than 1, it represents that only a fraction of trips use the EV sharing service. Otherwise if this factor is greater than 1 , the increased demand can be interpreted as a result of future population growth in this area. Figures $5(\mathrm{~g})$ and $(\mathrm{h})$ show that as the trip inflation factor increases, all cost components increase, which is intuitive because more demands require higher service investment. Note that the corresponding sensitivity factors are all less than 10 (the ratio of the the two inflation factor values at which the sensitivity factor is calculated), and thus we see that economies of scale do exist in the system.

After the sensitivity analysis, we will examine the optimal system design. Figure 6 plots the optimal station locations (the colored dots), their service partitions (the polygons with blue edges) and the corresponding initial fleet sizes $I(x)$ (color scales specified by the color bar). We see that the geometries of most partitions are not too much different from a regular shape, which conforms with the assumption of regular partition shapes in CA. The initial fleet size of a station varies across a wide range, and its distribution is roughly consistent with that of net trip production rate shown in Figure 2 (c). This suggests stations in areas where the produced trips far exceed attracted ones likely need a large $I(x)$, whereas stations in areas with negative net productions can just keep a low $I(x)$, which will nonetheless be replenished by dominating arriving trips to a sufficient level. Further, we see that the partition sizes in areas with considerably low or high net productions are in general smaller that those in areas with mid-range net productions. For a partition with an extremely low (or negative) net production rate, $I(x)$ is already close to zero regardless of the partition size, and thus there is little pooling effect that can bring down the unit-area fleet size by aggregating trips (or increasing the partition size). This probably explains why the partition size is not large for areas with very low production rates. On the contrary, for an area with very high net production rate, by comparing Figures 2 (a), (b) and (c), we note that such an area usually has relatively high trip production and attraction rates, and thus the partition size also needs to be small so as to keep the total walking cost low.

Figures 7 and 8 show the system designs when one parameter becomes one fifth of or two times its default value (and the others remain at their default values). We choose to vary $f, w, h$ and the trip inflation factor, because Figures 4 and 5 suggest that the cost components are relatively sensitive to these parameters. Figures 7 (a) and (b) show that as $f$ goes up, the sizes of most partitions increase, and interestingly, the increases of the partitions in areas with negative net production rates (see Figure 2 (c)) seem to be more than those in other areas. This is probably because that the total cost of a partition with a high trip production rate is dominated by the fleet holding cost and thus is less affected by the station investment. As the partition size increases, the magnitude of $I(x)$ increases as well. As plotted in Figures 7 (c) and (d), when $w$ is small or walking is less bothering to the travelers, partition sizes are large and $I(x)$ values are high, whereas when walking is expensive, the stations are very densely distributed, each holding much fewer EVs. Figures 8 (a) and (b) show that as $h$ increases, the partition sizes increase, and the increases of partitions in areas with high net production rates seem to be more dramatic. This is again probably 


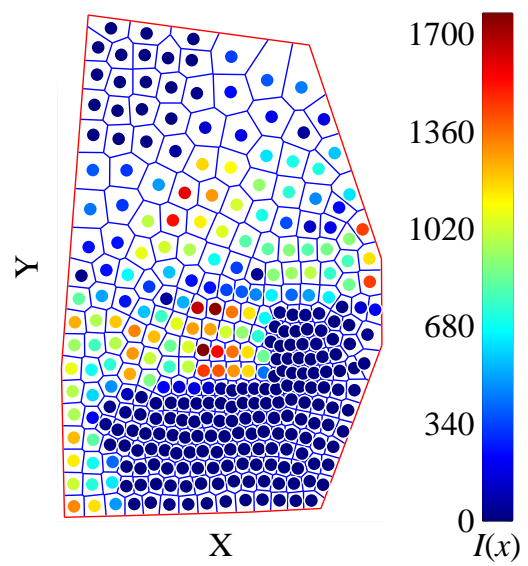

Figure 6: Optimal station locations and corresponding EV fleet sizes with the default parameters.

because that the total costs in areas with high trip production rates are dominated by $h$ and thus the change of the $h$ value will impact these areas more. Figures 8 (c) and (d) show that as the trip inflation factor increases (or the total population using the EV sharing service grows), all partitions shrink almost proportionally.

We further test our model and algorithm in a large metropolitan network: Chicago Sketch Network, also prepared by Dr. Bar-Gera (http://www.bgu.ac.il/ ${ }^{b}$ argera/tntp/). The purpose is to investigate the model performance on large scale networks, and the effectiveness of the EV sharing design model is proved. Similar insights are also obtained from sensitivity analysis. Please see Appendix $\mathrm{C}$ for detailed results and design plots.

\section{Conclusion}

This paper closes the research gap in the literature by developing a comprehensive design framework on how to economically deploy a one-way EV car sharing system that is able to provide reliable service to stochastic trip demands in an urban area. This paper studies the optimal design of such an EV car sharing system, including the location of car sharing stations and the corresponding vehicle inventories at these stations under stochastic demand and EV charging constraints, to minimize the comprehensive system cost (including station construction investment, vehicle maintenance, transportation, and vehicle balancing). This problem is NP-hard and deals with densely distributed charging facilities, numerous EVs with a non-linear charging capacity and massive stochastic and dynamic trips. We formulate this problem in a continuous model and propose a CA approach to solve this model. We show that this CA approach can overcome these modeling challenges by decomposing the studied area into small neighborhoods that each can be approximated by an Infinite Homogeneous Plane (IHP). We find that the system cost of an IHP is a unimodal function of the facility service area size and can be efficiently solved in a sub-linear time by the bisection algorithm. Then integrating the solutions of all IHPs yields an approximated solution to the original heterogeneous area. Numerical experiments show that the CA solution is able to estimate the total system cost of the discrete counterpart solution very efficiently with reasonable accuracy, even for relatively heterogeneous problems. Managerial insights into the approximation accuracy and parameter sensitivities are drawn from these numerical results. Overall, the proposed mod- 
(a) $f=\$ 100 /$ station

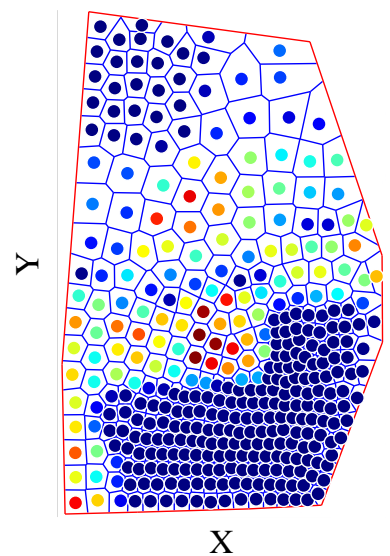

(c) $w=\$ 1.25 / \mathrm{km}$

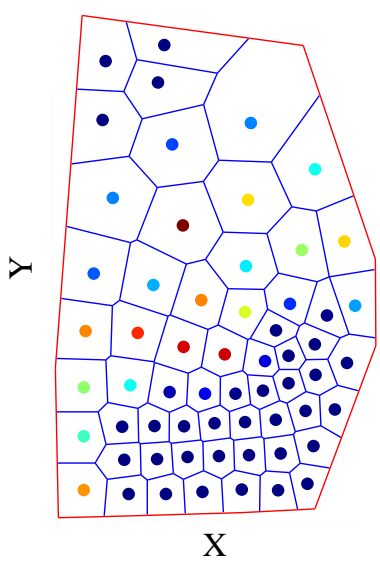

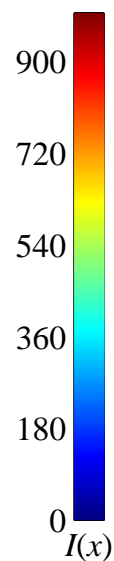

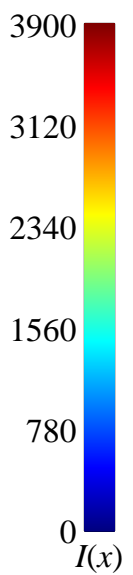

(b) $f=\$ 1000 /$ station
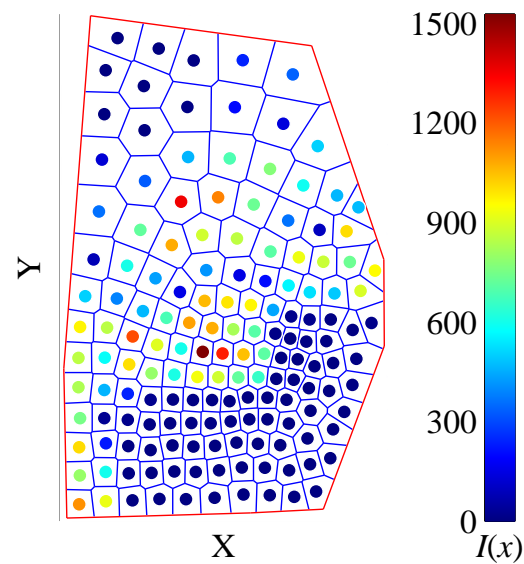

(d) $w=\$ 12.5 / \mathrm{km}$

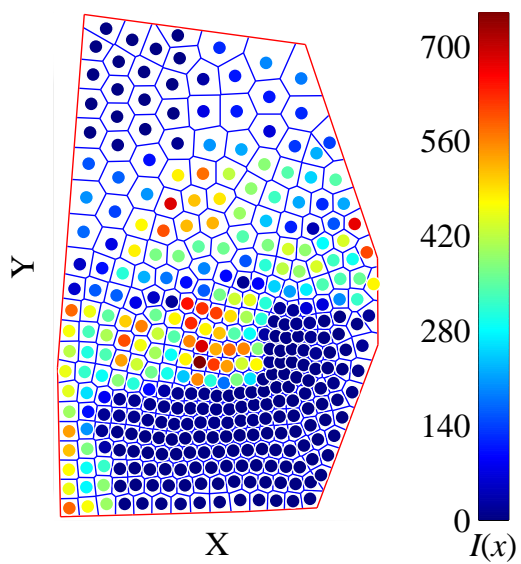

Figure 7: Optimal station locations and corresponding EV fleet sizes when $f$ or $w$ varies. 

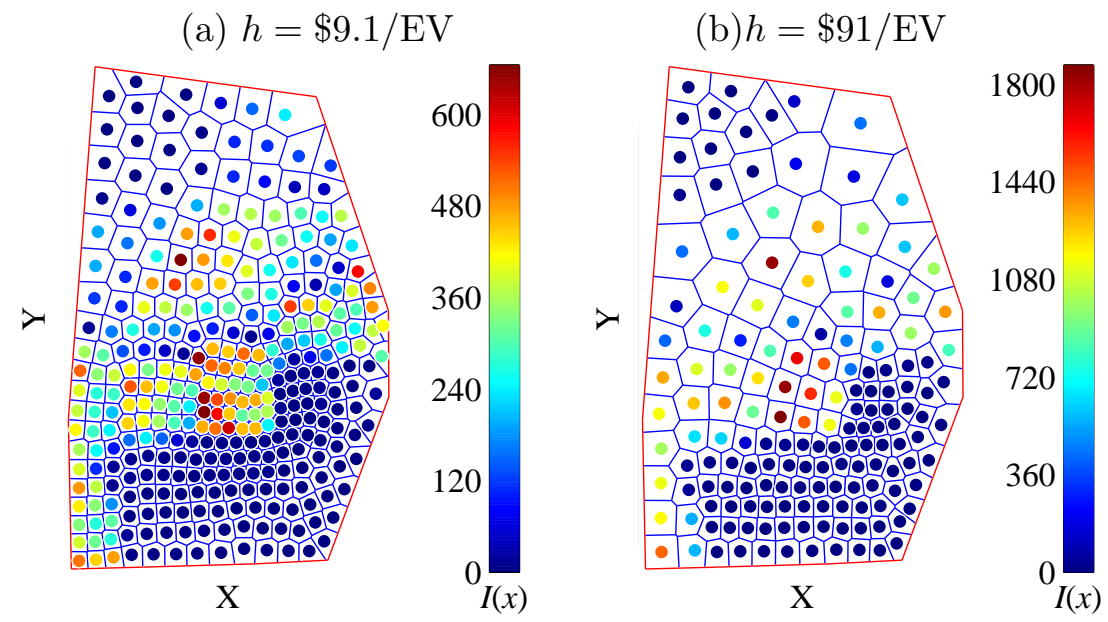

(c) Trip inflation factor $=0.2$
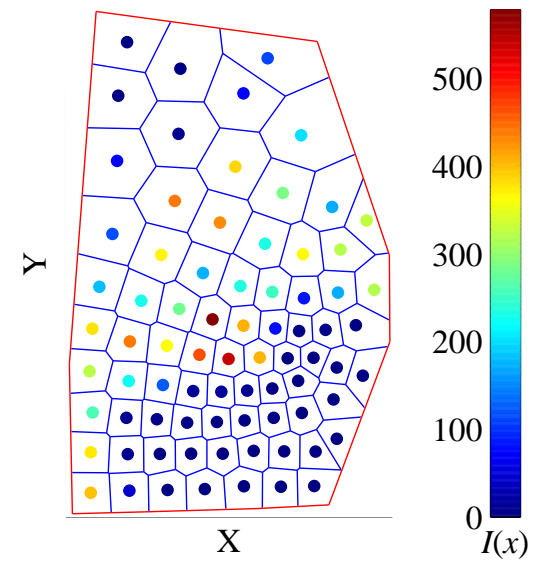

(d)Trip inflation factor $=2$

Figure 8: Optimal station locations and corresponding EV fleet sizes when $h$ or the trip inflation factor varies. 
eling framework serves as a methodology foundation for expanding existing infrastructure and for advancing operational paradigms of EV sharing systems. It will benefit the development of future sustainable transportation systems that best support economy, environment and social welfare.

We would note that the proposed EV sharing framework makes a few key assumptions: 1) the trip rate $\gamma\left(x^{\prime}, y^{\prime}, t\right)$ values are dense (and thus the EV sharing facilities are dense and walking distance is relatively small compared to driving distance) and independent temporally and spatially; 2) an EV sharing facility has sufficient charging capacity such that an inbound EV is connected to a charger as soon as it is dropped off; 3) an outbound EV is available for pickup only when it is fully charged for protecting the battery; 4) an EV always travels at a constant speed $v$ without being affected by traffic congestion; and 5) the cost of moving an EV is fixed regardless of the moving distance. While these assumptions allow us to simplify the analysis and capture fundamental elements in planning a large-scale EV sharing system, they may not fit all customized applications. When this framework is applied to engineering problems, further studies may be needed to relax certain assumptions according to the specific settings.

This research also opens up a number of future research opportunities. It will be very interesting to investigate how to increase the accuracy of CA approximation with acceptable computational complexity. Based on the decomposition scheme in the CA approach, parallel or distributed computing methods may be adopted to enhance the solution efficiency, which shall be particularly valuable for EV sharing system design in mega cities with large populations. This fundamental study makes a number of assumptions (e.g., full charge before departure, fixed arrival time, sufficient charging capacity) and approximations (e.g., using direct OD distance to approximate hub-to-hub distances) in the system operations. High fidelity models can be developed in the future to relax these assumptions and approximations. Further, it is interesting to coordinate an EV sharing system with other transportation modes to better distribute demands among multiple modes and to promote proper intermodal services (e.g., park-and-ride). This multi-modal perspective can further improve both the transportation system overall performance and each individual's experience with it.

\section{Acknowledgments}

This research is supported in part by the U.S. National Science Foundation through Grants CMMI \#1558889 and CMMI \#1541130 and by the National Center for Intermodal Transportation for Economic Competitiveness (a Tier-I University Transportation Center) through Grant DTRT12G-UTC14-364803.

\section{References}

Axhausen, K., Polak, J., Boltze, M., Puzicha, J., 1994. Effectiveness of the parking guidance system in frankfurt/main. Traffic Engineering and Control 35 (5), 304-309.

Barth, M., Todd, M., 1999. Simulation model performance analysis of a multiple station shared vehicle system. Transportation Research Part C: Emerging Technologies 7 (4), 237-259.

Berman, O., Bertsimas, D., Larson, R. C., 1995. Locating discretionary service facilities, ii: maximizing market size, minimizing inconvenience. Operations Research 43 (4), 623-632. 
Berman, O., Larson, R. C., Fouska, N., 1992. Optimal location of discretionary service facilities. Transportation Science 26 (3), 201-211.

Britton, E., 2000. Carsharing 2000: Sustainable transport's missing link. Eco-Logica.

Bruglieri, M., Colorni, A., Luè, A., 2014. The vehicle relocation problem for the one-way electric vehicle sharing: An application to the milan case. Procedia-Social and Behavioral Sciences 111, $18-27$.

Bureau, o. T. S., 2014. Pocket guide to transportation. US Department of Transportation, Bureau of Transportation Statistics, Washington.

Campbell, J. F., 1993a. Continuous and discrete demand hub location problems. Transportation Research Part B: Methodological 27 (6), 473-482.

Campbell, J. F., 1993b. One-to-many distribution with transshipments: An analytic model. Transportation Science 27 (4), 330-340.

Carlsson, J. G., Jia, F., 2013. Euclidean hub-and-spoke networks. Operations Research 6 (61), 1360 $-1382$.

Carlsson, J. G., Jia, F., Li, Y., 2013. An approximation algorithm for the continuous k-medians problem in a convex polygon. INFORMS Journal on Computing 2 (26), $280-289$.

Chakirov, A., Fourie, P., 2013. Enriched sioux falls scenario with dynamic and disaggregate demand. Tech. rep., Technical report, Future Cities Laboratory, Singapore-ETH Centre (SEC), 2014. www. vsp. tu-berlin. de/publications.

Chauvet, F., PROTH, J.-M., SAUER, N., et al., 1997. Management of a pool of self-service cars. Journal of Intelligent Manufacturing 8 (5), 459-465.

Chemla, D., M. F., Calvo, R. W., 2013. Bike sharing systems: Solving the static rebalancing problem. Discrete Optimization 10 (2), 120-146.

Chen, L.-R., 2007. A design of an optimal battery pulse charge system by frequency-varied technique. Industrial Electronics, IEEE Transactions on 54 (1), 398-405.

Chen, Q., Li, X., Ouyang, Y., 2011. Joint inventory-location problem under the risk of probabilistic facility disruptions. Transportation Research Part B 45 (7), 991 - 1003.

Ciari, F., Balmer, M., Axhausen, K. W., Axhausen, K. W., Axhausen, K. W., 2008. Concepts for a large scale car-sharing system: Modeling and evaluation with an agent-based approach. Eidgenössische Technische Hochschule, Institut für Verkehrsplanung und Transportsysteme.

Cooper, G., Howe, D. A., Mye, P., 2000. The missing link: An evaluation of carsharing Portland Inc., Portland, Oregon.

Correia, G. H. d. A., Antunes, A. P., 2012. Optimization approach to depot location and trip selection in one-way carsharing systems. Transportation Research Part E: Logistics and Transportation Review 48 (1), 233-247. 
Cui, T., Ouyang, Y., Shen, Z. M., 2010. Reliable facility location under the risk of disruptions. Operations Research 58 (4), 998-1011.

Daganzo, C., Newell, G., 1986. Configuration of physical distribution networks. Networks 16 (2), $113-132$.

Daganzo, C. F., 2005. Logistics systems analysis. Springer.

Daganzo, C. F., Erera, A. L., 1999. On planning and design of logistics systems for uncertain environments. In: New Trends in Distribution Logistics. Springer, pp. 3-21.

Daskin, M., 1995. Network and Discrete Location: Models, Algorithms, and Applications. John Wiley, New York.

Delucchi, M. A., Lipman, T. E., 2001. An analysis of the retail and lifecycle cost of battery-powered electric vehicles. Transportation Research Part D: Transport and Environment 6 (6), 371-404.

Drezner, Z. (Ed.), 2002. Facility Location: A Survey of Applications and Methods. Springer, New York.

Du, Y., Hall, R., 1997. Fleet sizing and empty equipment redistribution for center-terminal transportation networks. Management Science 43 (2), 145-157.

Fan, W. D., Machemehl, R. B., Lownes, N. E., 2008. Carsharing: Dynamic decision-making problem for vehicle allocation. Transportation Research Record: Journal of the Transportation Research Board 2063 (1), 97-104.

George, D. K., Xia, C. H., 2011. Fleet-sizing and service availability for a vehicle rental system via closed queueing networks. European Journal of Operational Research 211 (1), 198-207.

Green, C., 2009. Car-sharing - good for the environment and the budget. http://www.blueplanetgreenliving.com/2009/09/16/ car-sharing-good-for-the-environment-and-the-budget/, accessed on Jan/30/2016.

Hall, R. W., 1989. Configuration of an overnight package air network. Transportation Research Part A: General 23 (2), 139-149.

He, F., Wu, D., Yin, Y., Guan, Y., 2013. Optimal deployment of public charging stations for plug-in hybrid electric vehicles. Transportation Research Part B: Methodological 47, 87-101.

He, F., Yin, Y., Lawphongpanich, S., 2014. Network equilibrium models with battery electric vehicles. Transportation Research Part B: Methodological 67, 306-319.

Hodgson, M. J., 1990. A flow-capturing location-allocation model. Geographical Analysis 22 (3), 270-279.

Huang, Y., Li, S., Qian, Z. S., 2015. Optimal deployment of alternative fueling stations on transportation networks considering deviation paths. Network and Spatial Economics 15 (1), 183-204.

Ip, A., Fong, S., Liu, E., 2010. Optimization for allocating bev recharging stations in urban areas by using hierarchical clustering. In: Advanced Information Management and Service (IMS), 2010 6th International Conference on. IEEE, pp. 460-465. 
Jakle, J. A., Sculle, K. A., 2004. Lots of parking: Land use in a car culture. University of Virginia Press.

Kansas Department of Transportation, 2009. 5-county regional transportation study phase 1 final report. http://kdotapp.ksdot.org/5CountyStudy/get_more_info/reports.aspx, accessed on Jan/30/2016.

Kek, A. G., Cheu, R. L., Meng, Q., Fung, C. H., 2009. A decision support system for vehicle relocation operations in carsharing systems. Transportation Research Part E: Logistics and Transportation Review 45 (1), 149-158.

King, C., Griggs, W., Wirth, F., Shorten, R., 2013. Using a car sharing model to alleviate electric vehicle range anxiety. In: he 16th Yale Workshop on Adaptive and Learning Systems. pp. 130135.

Kuby, M., Lim, S., 2005. The flow-refueling location problem for alternative-fuel vehicles. SocioEconomic Planning Sciences 39 (2), 125-145.

Kuby, M., Lines, L., Schultz, R., Xie, Z., Kim, J.-G., Lim, S., 2009. Optimization of hydrogen stations in florida using the flow-refueling location model. International journal of hydrogen energy 34 (15), 6045-6064.

Kumar, V. P., Bierlaire, M., 2012. Optimizing locations for a vehicle sharing system. In: Swiss Transport Research Conference. http://www.strc.ch/conferences/2012/Kumar_Bierlaire. pdf, accessed on Jan/30/2016.

Langevin, A., M. P. . C. J. F., 1996. Continuous approximation models in freight distribution: An overview. Transportation Research Part B: Methodological 3 (30), 163 - 188.

Li, X., 2015. A linear-time sweeping algorithm for discretization of continuum approximation. Working paper, University of South Florida.

Li, X., Ouyang, Y., 2010. A continuum approximation approach to reliable facility location design under correlated probabilistic disruptions. Transportation Research Part B 44 (4), 535-548.

Litman, T., 2007. Parking management: Comprehensive implementation guide. VTPI (www. vtpi. org).

Love, R. F., Morris, J. G., 1979. Mathematical models of road travel distances. Management Science 25 (2), 130-139.

Mak, H.-Y., Rong, Y., Shen, Z.-J. M., 2013. Infrastructure planning for electric vehicles with battery swapping. Management Science 59 (7), 1557-1575.

Milgrom, P., Segal, I., 2002. Envelope theorems for arbitrary choice sets. Econometrica 70 (2), 583-601.

Millard-Ball, A., 2005. Car-Sharing: Where and how it succeeds. Vol. 108. Transportation Research Board. 
Nie, Y. M., Ghamami, M., 2013. A corridor-centric approach to planning electric vehicle charging infrastructure. Transportation Research Part B: Methodological 57, 172-190.

Nobis, C., 2006. Carsharing as key contribution to multimodal and sustainable mobility behavior: Carsharing in germany. Transportation Research Record: Journal of the Transportation Research Board 1986 (1), 89-97.

Nourbakhsh, S. M., Ouyang, Y., 2010. Optimal fueling strategies for locomotive fleets in railroad networks. Transportation Research Part B: Methodological 44 (8), 1104-1114.

Okabe, A., Boots, B., Sugihara, K., Chiu, S. N., 2009. Spatial tessellations: concepts and applications of Voronoi diagrams. Vol. 501. John Wiley \& Sons.

Ouyang, Y., . D. C. F., 2006. Discretization and validation of the continuum approximation scheme for terminal system design. Transportation Science 40 (1), 89 - 98.

Ouyang, Y., Nourbakhsh, S. M., Cassidy, M. J., 2014. Continuum approximation approach to bus network design under spatially heterogeneous demand. Transportation Research Part B: Methodological 68 (3), $333-344$.

Ouyang, Y., Wang, Z., Yang, H., 2015. Facility location design under continuous traffic equilibrium. Transportation Research Part B: Methodological 81 (1), 18 - 33.

Pal, A., Zhang, Y., 2016. Free-floating bike sharing: Solving real-life large-scale static rebalancing problems. Computers and Operations Research,under review.

Pan, F., Bent, R., Berscheid, A., Izraelevitz, D., 2010. Locating phev exchange stations in v2g. In: Smart Grid Communications (SmartGridComm), 2010 First IEEE International Conference on. IEEE, pp. 173-178.

Qureshi, M. A., Hwang, H.-L., Chin, S.-M., 2002. Comparison of distance estimates for commodity flow survey: Great circle distances versus network-based distances. Transportation Research Record: Journal of the Transportation Research Board 1804 (1), 212-216.

Regue, R., Recker, W., 2014. Proactive vehicle routing with inferred demand to solve the bikesharing rebalancing problem. Transportation Research Part E: Logistics and Transportation Review 72, $192-209$.

Shaheen, P., Cohen, A., 2013. Innovative mobility carsharing outlook. University of Berkeley, California.

Shaheen, S., Sperling, D., Wagner, C., 1998. Carsharing in europe and north american: Past, present, and future. Transportation Quarterly 52 (3), 35-52.

Shen, Z.-J. M., Coullard, C., Daskin, M. S., 2003. A joint location-inventory model. Transportation Science 37 (1), 40-55.

Sinha, K. C., 2003. Sustainability and urban public transportation. Journal of Transportation Engineering 129 (4), 331-341. 
Smith, S. L., Pavone, M., Schwager, M., Frazzoli, E., Rus, D., June, 2013. Rebalancing the rebalancers: Optimally routing vehicles and drivers in mobility-on-demand systems. In: IEEE American Control Conference (ACC). pp. 2362-2367.

Sweda, T., Klabjan, D., 2011. An agent-based decision support system for electric vehicle charging infrastructure deployment. In: Vehicle Power and Propulsion Conference (VPPC), 2011 IEEE. IEEE, pp. 1-5.

Tirachini, A., Hensher, D. A., Rose, J. M., 2014. Multimodal pricing and optimal design of urban public transport: The interplay between traffic congestion and bus crowding. Transportation Research Part B: Methodological 61, 33-54.

Toh, R. S., Higgins, R. G., 1985. The impact of hub and spoke network centralization and route monopoly on domestic airline profitability. Transportation Journal 24 (4), 16-27.

Tsao, Y.-C., Mangotra, D., Lu, J.-C., Dong, M., 2012. A continuous approximation approach for the integrated facility-inventory allocation problem. European journal of operational research $222(2), 216-228$.

Uesugi, K., Mukai, N., Watanabe, T., 2007. Optimization of vehicle assignment for car sharing system. In: Knowledge-based intelligent information and engineering systems. Springer, pp. 11051111.

Upchurch, C., Kuby, M., Lim, S., 2009. A model for location of capacitated alternative-fuel stations. Geographical Analysis 41 (1), 85-106.

Valdes-Dapena, P., 2008. GM unveils chevy volt - looking to the future. http://money. cnn.com/galleries/2008/autos/0809/gallery.gm_volt_reveal/index.html, accessed on $\operatorname{Jan} / 30 / 2016$.

Vlasic, B., 2013. 2 makers press the case for electric cars. http://www.theterranews.com/ content/?p=567561, accessed on Jan/30/2016.

Wang, M., Weber, T., Darlington, T., 2005. Well-to-wheels analysis of advanced fuel/vehicle systems: A north american study of energy use, greenhouse gas emissions, and criteria pollutant emissions. http://energy.gov/eere/bioenergy/downloads/ well-wheels-analysis-advanced-fuelvehicle-systems-north-american-study, accessed on Jan/30/2016. Department of Energy.

Wang, X., Ouyang, Y., 2013. A continuum approximation approach to competitive facility location design under facility disruption risks. Transportation Research Part B: Methodological 50, 90103.

Wang, Y.-W., Lin, C.-C., 2009. Locating road-vehicle refueling stations. Transportation Research Part E: Logistics and Transportation Review 45 (5), 821-829.

Yilmaz, M., Krein, P. T., 2012. Review of charging power levels and infrastructure for plug-in electric and hybrid vehicles. In: Electric Vehicle Conference (IEVC), 2012 IEEE International. IEEE, pp. 1-8. 


\section{Appendix}

\section{A Notation}

The following table lists all key parameters and variables used in this paper.

\section{B Proofs of Lemmas and Propositions in Section 4.1}

The Appendix B includes the proofs for the Lemmas and Propositions in Section 4.1.

Lemma 4.1. Function $C(A)$ is continuous, non-negative and $\lim _{A \rightarrow 0^{+}} C(A)=\infty, \lim _{A \rightarrow \infty} C(A)=$ $\infty$.

Proof. The continuity and non-negativity can be directly obtained from such properties of each term in formula (17). Further, term $f A^{-1}+w(\bar{\lambda}(\bar{t})+\bar{\mu}(\bar{t})) \eta g A^{0.5}$ goes to infinity as $A$ approaches to either 0 or $\infty$. Since both $C_{h}(A)$ and $C_{b}(A)$ are non-negative $\forall A \in(0, \infty)$, it is obvious that $\lim _{A \rightarrow 0^{+}} C(A)=\infty, \lim _{A \rightarrow \infty} C(A)=\infty$.

Lemma 4.2. There exists a finite $A^{*} \in(0, \infty)$ such that $C\left(A^{*}\right)=C^{*}$ and $0 \in \dot{C}\left(A^{*}\right)$.

Proof. Lemma 4.1 indicates that $C(A)$ has a finite minimizer $A^{*}$. Since $C(A)$ is continuous, $\dot{C}\left(A^{*}\right)$ exists and should contain sub-gradient 0 .

Proposition 4.1. Define $G(A):=I(A) A^{-1}$. If $\dot{G}(A) A^{\delta}$ is increasing over $A \in(0, \infty)$ for some $\delta \in[0.5,1.5]$, there exists a $A^{*} \in(0, \infty)$ such that $0 \in \dot{C}\left(A^{*}\right), \dot{C}(A)<0, \forall A \in\left(0, A^{*}\right)$ and $\dot{C}(A)>0, \forall A \in\left(A^{*}, \infty\right)$; i.e., function $C(A)$ strictly unimodal with a unique minimizer of $A^{*} \in(0, \infty)$.

Proof. Further we define $G_{\bar{t}}(A):=I_{\bar{t}}(A) A^{-1}$. Based on formula (17), we obtain

$$
\dot{C}(A) A^{\delta}=-f A^{\delta-2}+0.5 w(\bar{\lambda}(\bar{t})+\bar{\mu}(\bar{t})) \eta g A^{\delta-0.5}+h \dot{G}(A) A^{\delta}+b \dot{G}_{\bar{t}}(A) A^{\delta}
$$

Note that term $-f A^{\delta-2}+0.5 g w \eta(\bar{\lambda}(\bar{t})+\bar{\mu}(\bar{t})) A^{\delta-0.5}$ is strictly increasing with $A \in(0, \infty)$ for any $\delta \in[0,1.5]$. Further, we obtain

$$
\dot{G}_{\bar{t}}(A) A^{\delta}=-A^{\delta-1.5} \frac{\hat{\theta}(\bar{t})}{2} \phi\left(\frac{\bar{\theta}(\bar{t}) A^{0.5}}{\hat{\theta}(\bar{t})}\right) .
$$

From the above equation, it is easy to see that term $b \dot{G}_{\bar{t}}(A) A^{\delta}$ increases with $A$ as well given $\delta \leq 1.5$. Since $\dot{G}(A) A^{\delta}$ is strictly increasing over $A \in(0, \infty)$ as well, then we obtain $\dot{C}(A) A^{\delta}$ is strictly increasing over $A \in(0, \infty)$ as well. From lemma 4.2 , we know that there exists a $A^{*}$ such that $0 \in \dot{C}\left(A^{*}\right)$, and thus $0 \in \dot{C}\left(A^{*}\right) A^{\delta}$ too. Then since $\dot{C}(A) A^{\delta}$ is strictly increasing almost everywhere, we obtain $\dot{C}(A) A^{\delta}<0, \forall A \in\left(0, A^{*}\right)$ and $\dot{C}(A) A^{\delta}>0, \forall A \in\left(A^{*}, \infty\right)$. Removing the positive scalar $A^{\delta}$ would not affect the sign of these inequalities, this implies that $C(A)$ is strictly decreasing $\forall A \in\left(0, A^{*}\right)$ and strictly increasing $\forall A \in\left(A^{*}, \infty\right)$. This completes the proof. 
Table 4: Notation for key variables and parameters

\begin{tabular}{|c|c|}
\hline $\begin{array}{l}\text { Variables and } \\
\text { Parameters }\end{array}$ & Notes \\
\hline$T$ & time period; we consider $[0, \bar{t}]$ in this study \\
\hline $\mathbf{x}$ & $\begin{array}{l}\text { location vector for EV sharing facilities } \mathbf{x}:=\left\{x_{1}, x_{2}, \ldots, x_{N}\right\} \text { on } \\
\text { two-dimensional space } \mathcal{X} \subseteq \mathbb{R}^{2}\end{array}$ \\
\hline$f(x)$ & unit-cycle pro-rated construction cost at location $x$ \\
\hline $\mathcal{X}_{\mathbf{x}}(x)$ & set of locations in the partition with seed (or facility) $x$ \\
\hline$S_{\mathbf{x}}(x)$ & seed for any location $x \in \mathcal{X}$ \\
\hline$\lambda(x, t)$ & accumulative departure count at location $x$ and time $t$ \\
\hline$\mu(x, t)$ & accumulative arrival count at location $x$ and time $t$ \\
\hline$\theta(x, t)$ & cumulative net departure count at location $x$ and time $t$ \\
\hline$\gamma^{+}(x, t)$ & randomly generated trip rate per unit area at location $x$ and time $t$ \\
\hline$\gamma^{-}(x, t, s)$ & $\begin{array}{l}\text { random rate of arriving EVs at the SOC of } s \text { per unit area around } \\
\text { location } x \text { at time } t\end{array}$ \\
\hline$s$ & state of charge (SOC) $s \in[0, \bar{s}]$ \\
\hline$h$ & inventory cost for maintaining an EV per cycle \\
\hline$f$ & cost to build a EV sharing facility \\
\hline$g$ & $\begin{array}{l}\text { unit-distance transportation cost; (CA) distance between the centroid } \\
\text { and an average point in a regular hexagon of area size of } 1\end{array}$ \\
\hline$b$ & rebalancing cost per car \\
\hline$v$ & average vehicle operation speed \\
\hline$I(x)$ & number of EVs at the beginning time 0 at location $x$ \\
\hline$H(s)$ & denote the time to charge an EV at SOC $\bar{s}-s$ to full SOC $\bar{s}$ \\
\hline$C_{f}$ & total cost of station construction \\
\hline$C_{w}$ & total cost of walking cost by all travelers \\
\hline$C_{h}$ & total cost of maintaining all EVs in the system \\
\hline$C_{b}$ & total cost of rebalancing EVs between facilities \\
\hline$A$ & $\begin{array}{l}\text { the partition area size of a hexagon covered by a facility on an infinite } \\
\text { homogeneous plane (IHP) in CA analysis. }\end{array}$ \\
\hline$\triangle l$ & width of equal-area unit squares $q \in \mathcal{Q}$ with a central coordinate of $x_{q}$. \\
\hline$\triangle t$ & small time interval separated by discretized time points. \\
\hline
\end{tabular}


Proposition 4.2. If $I(A)=I^{F}(A)$ (i.e., when the FTA criterion is adopted), $C(A)$ is strictly unimodal with a unique minimizer over $A \in(0, \infty)$.

Proof. From formula (14), we obtain $G(A)=\left[\bar{\theta}(\hat{t})+\Phi^{-1}(\alpha) \hat{\theta}(\hat{t}) A^{-0.5}\right]^{+}$. If $\bar{\theta}(\hat{t}) \geq 0$, we obtain $\dot{G}(A) A^{0.5}=-0.5 \hat{\theta}(\bar{t}) \Phi^{-1}(\alpha) A^{-1}$, which is obviously increasing with $A \in(0, \infty)$. Otherwise if $\bar{\theta}(\hat{t})<0$,

$$
\dot{G}(A) A^{0.5}= \begin{cases}-0.5 \hat{\theta}(\bar{t}) \Phi^{-1}(\alpha) A^{-1}, & \text { if } 0<A<\frac{\Phi^{-1}(\alpha)^{2} \hat{\theta}(\hat{t})^{2}}{\bar{\theta}(\hat{t})^{2}} \\ \left\{-0.5 \hat{\theta}(\bar{t}) \Phi^{-1}(\alpha) A^{-1}, 0\right\}, & \text { if } A=\frac{\Phi^{-1}(\alpha)^{2} \hat{\theta}(\hat{t})^{2}}{\bar{\theta}(\hat{t})^{2}} \\ 0 & \text { if } A>\frac{\Phi^{-1}(\alpha)^{2} \hat{\theta}(\hat{t})^{2}}{\bar{\theta}(\hat{t})^{2}}\end{cases}
$$

and therefore we see that $\dot{G}(A) A^{0.5}$ increases with with $A \in(0, \infty)$ as well. Then Proposition 4.1 tells that $C(A)$ is strictly unimodal with a unique minimizer.

Proposition 4.3. If $I(A)=I^{P}(A)$ (i.e., when the PTA criterion is adopted), $C(A)$ is strictly unimodal with a unique minimizer over $A \in(0, \infty)$.

Proof. We first define

$$
G(t, A):=\bar{\theta}(t)+\Phi^{-1}(\alpha) \hat{\theta}(t) A^{-0.5} .
$$

Then we can apply Theorem 3 in Milgrom and Segal (2002), a generalized envelope theory to prove this proposition. With this theorem, since functions $G(t, \cdot)_{t \in[0, T]}$ is apparently equidifferentiable at $A \in(0, \bar{A})$ where $\bar{A}$ is an arbitrarily large number, that $\sup _{t \in[0, T]} G(t, A)<\infty$ and that $T^{*}(A):=\arg \max _{t \in[0, \bar{t}]} G_{\epsilon}(t, A) \neq \emptyset$, then function $G(A):=\max _{t \in[0, \bar{t}]} G(t, A)$ is left- and right-hand differentiable at $A$, and for any $t^{*}(A) \in T^{*}(A)$, these directional derivatives can be formulated as

$$
\begin{aligned}
\dot{G}(A-) & :=\lim _{A^{\prime} \rightarrow A_{-}} \frac{\partial G\left(t^{*}\left(A^{\prime}\right), A\right)}{\partial A}=\lim _{A^{\prime} \rightarrow A^{-}}-\Phi^{-1}(\alpha) \bar{\sigma}\left(t^{*}\left(A^{\prime}\right)\right) A^{\prime-1.5} \\
\dot{G}(A+) & :=\lim _{A^{\prime} \rightarrow A+} \frac{\partial G\left(t^{*}\left(A^{\prime}\right), A\right)}{\partial A}=\lim _{A^{\prime} \rightarrow A+}-\Phi^{-1}(\alpha) \bar{\sigma}\left(t^{*}\left(A^{\prime}\right)\right) A^{\prime-1.5}
\end{aligned}
$$

We set $t^{*}(A)=\max T^{*}(A)$ if $\bar{\theta}(t)+\Phi^{-1}(\alpha) \hat{\theta}(t) A^{-0.5}>0$ for some $t$ or $t^{*}(A)=0$ if $\bar{\theta}(t)+$ $\Phi^{-1}(\alpha) \hat{\theta}(t) A^{-0.5} \leq 0, \forall t \in[0, \bar{t}]$. Since by definition $\hat{\theta}(t)$ increases with $t \in[0, T]$, based on the structure of equation $(21), t^{*}(A)$ shall be decreasing. Then from equations (22), we can see that both $\dot{G}(A+) A^{1.5}$ and $\dot{G}(A-) A^{1.5}$ are increasing with $A$. This is to say $\dot{G}(A) A^{1.5}$ is increasing with $A$. This combining Proposition 4.1 completes the proof.

\section{Chicago Sketch Network}

The network consists of 387 Origin-Destination zones and 933 auxiliary nodes, and is an aggregated yet fairly realistic representation of the Chicago region. The purpose of the large network test case is to understand the performance of the proposed model, algorithm efficiency and compare results with small test cases. The actual node coordinates are obtained through the same approach as in the Soux-Falls network. For the CA analysis, the border points of space $\mathcal{X}$ is created by expanding the convex hull of the 933 nodes along the radial direction from the centroid of the convex hull 
such that each corner point is $20 \%$ more distance from the centroid. The space is diced into unit squares of width $\Delta l=5 \mathrm{~km}$. This number is chosen considering the huge size of test network and the limitation of the experiment computer (16 GB RAM). Nonetheless, we believe this size is still reasonable considering relatively slow variations of parameter values within each unit square in a large area of Chicago network. The OD trips between the original 387 zones are interpolated across the whole space and then smoothed by the moving average method with a radius of $1 \mathrm{~km}$. We also remove the trips within a travel distance less than $\underline{d}=1 \mathrm{~km}$ that we assume will walk to their destinations. Trips longer than $\bar{s}$ (160 km for the base case) are assumed to use other types of transportation, and thus not considered in our analysis. It is also assumed that $10 \%$ of the total trips will rely on EVs in the whole Chicago network. The total number of original trips is 1202287 , and the total number of interpolated EV trips after removing short $(<1 \mathrm{~km})$ and long $(>160 \mathrm{~km})$ trips is 55725. We use the PTA criterion since it assures better service reliability.

We first examine the performance of CA approach and the discretization algorithm and their solution times. The parameters in Table 1 are used as benchmark instances, and we vary only one default parameter value at a time to investigate the sensitivity. The results are shown in Table 5. We find that, in addition to a few scenarios, the relative errors of the cost components between the CA approach and the discretization algorithm and their solution times are relatively small, with an average error of total cost $\bar{C}$ as $3.7 \%$. This confirms the previous conjecture that the CA methods works even better for larger-scale instances with less heterogeneous settings. Interestingly, the computational time is even smaller than the Sioux-Falls experiment, because there are fewer trips and only a slightly larger number of unit squares (due to larger $\Delta l$ ) are considered. In a few scenarios, however, the large $\Delta l$ value deviates the CA solutions from the discretization results in total walking cost $\bar{C}_{w}$. The relative errors of $\bar{C}_{w}$ are on average $11.6 \%$ with an maximum of $23.4 \%$. This is probably because the walking distance is small compared with $\Delta l$ and the approximation is not very accurate. Fortunately, the calculation of other cost components are tolerant to relatively large $\Delta l$, as evidenced by low relative errors in Table 5 . Therefore, the relative errors for total cost $\bar{C}$ remain small with an average of only $3.7 \%$ and a maximum of $11.4 \%$. This range of relative errors should be able to satisfy requirements of most engineering practices. We expect these errors can be significantly reduced if we further reduce $\Delta l$, which is however beyond the computation capacity of the available PC and is left for future research when computational resources are available.

The sensitivity analysis results are similar to the results obtained in the Sioux-Falls case study (Figure 4 and Figure 5) and thus we omit them in this presentation. We demonstrate the system designs for six different scenarios with varied key system parameters $(f, w$, and $h$ ), as shown in Figure 9. Note that we assume that $5 \%$ of the travelers will use the EV sharing for their travel, because location design plots become illegible when the demand are high. Figures 9 (a) and (b) show that as $f$ goes up, the sizes of most partitions increase. As the partition size increases, the magnitude of $I(x)$ increases as well. As plotted in Figures 9 (c) and (d), when $w$ is small or walking is less bothering to the travelers, partition sizes are larger and $I(x)$ values are higher. Whereas when walking is expensive, the stations are very densely distributed, each holding much fewer EVs. Figures 9 (e) and (f) show that as $h$ increases, the partition sizes increase and inventory $I(x)$ also increased. 


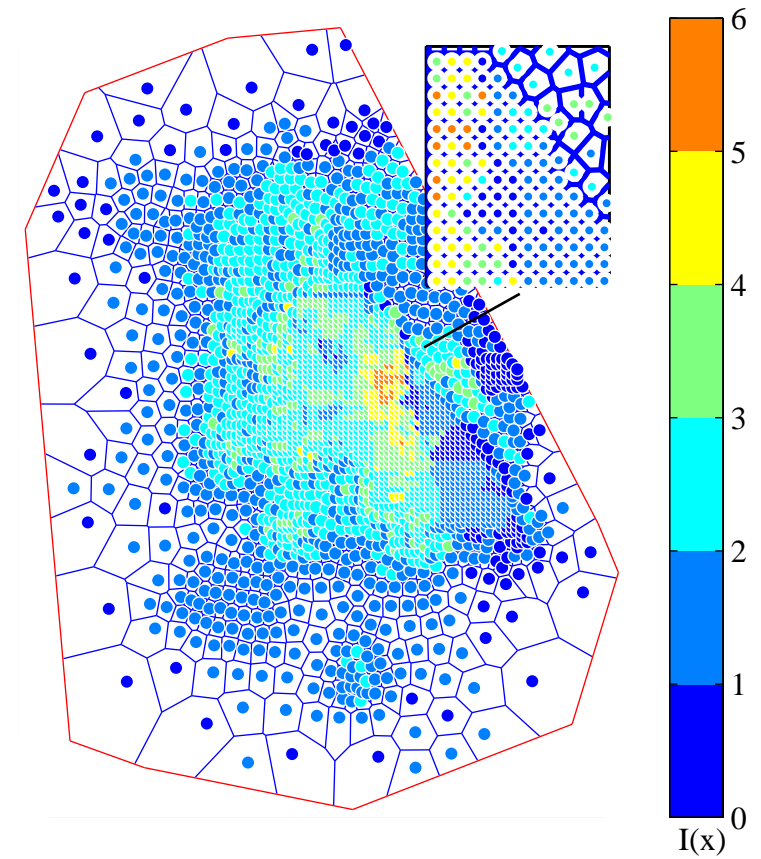

(a) $f=\$ 100 /$ station

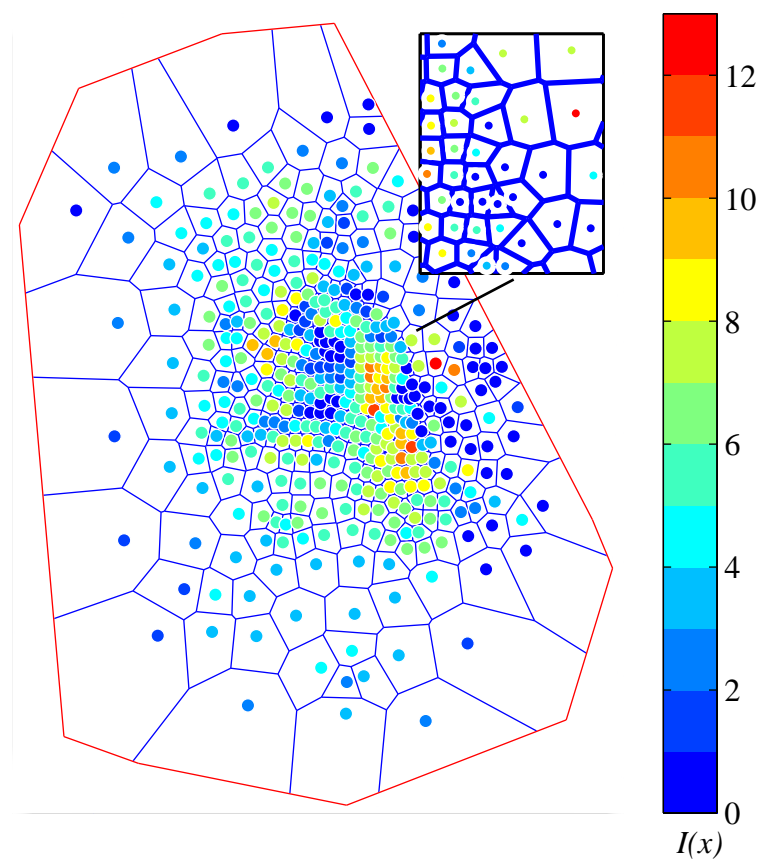

口

(b) $f=\$ 1000 /$ station

Figure 9: Chicago Case: Optimal station locations and corresponding EV fleet sizes when $f$. 


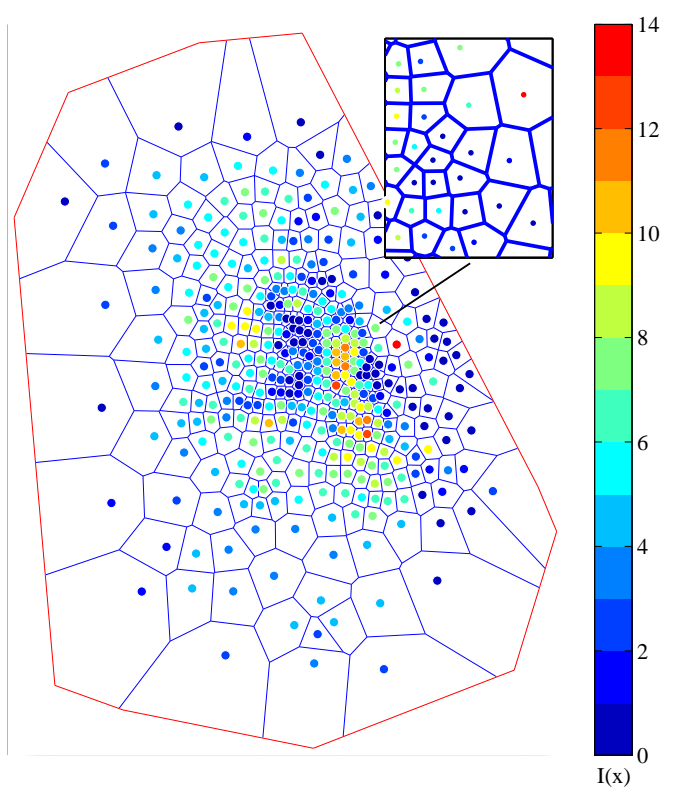

口

(a) $w=\$ 1.25 / \mathrm{km}$

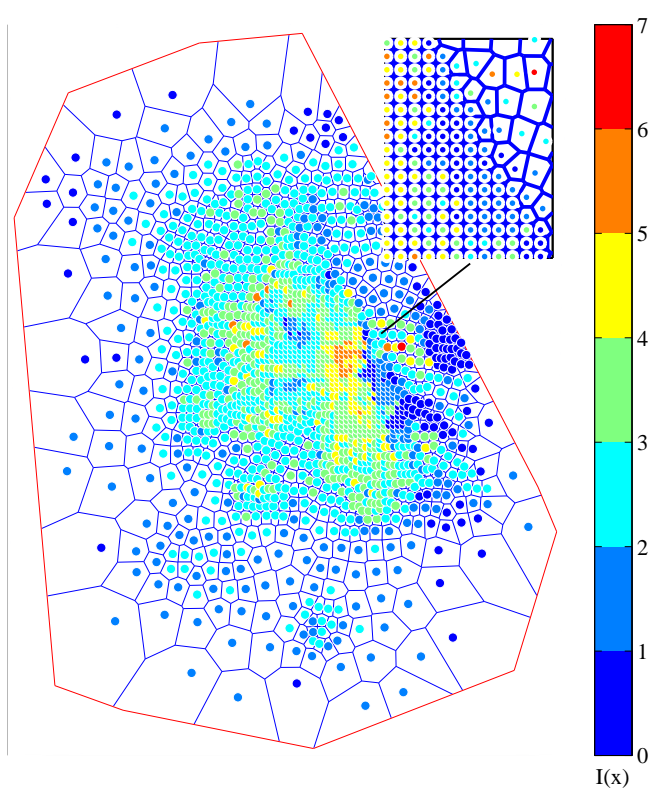

口

(b) $w=\$ 12.5 / \mathrm{km}$

Figure 10: Chicago Case: Optimal station locations and corresponding EV fleet sizes with varying $w$. 


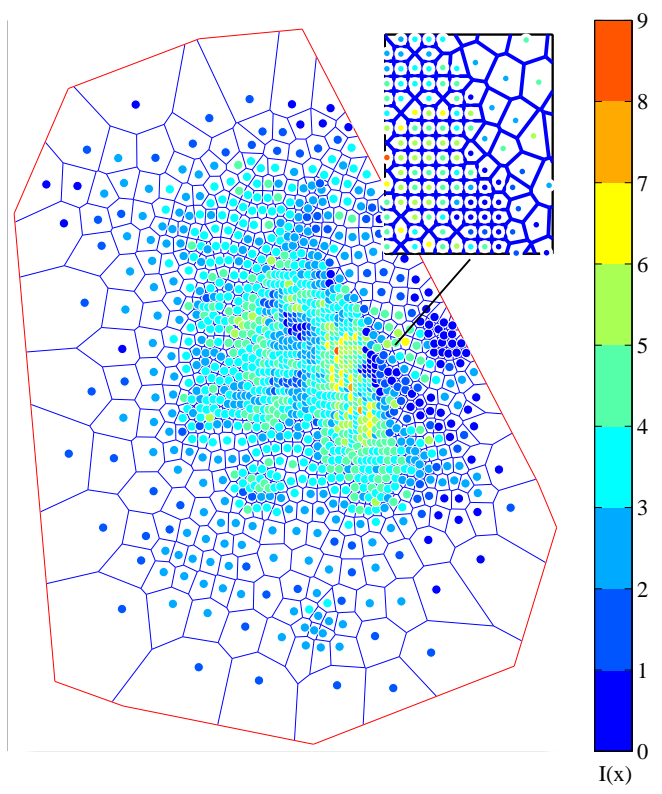

口

(a) $h=\$ 9.1 / \mathrm{EV}$

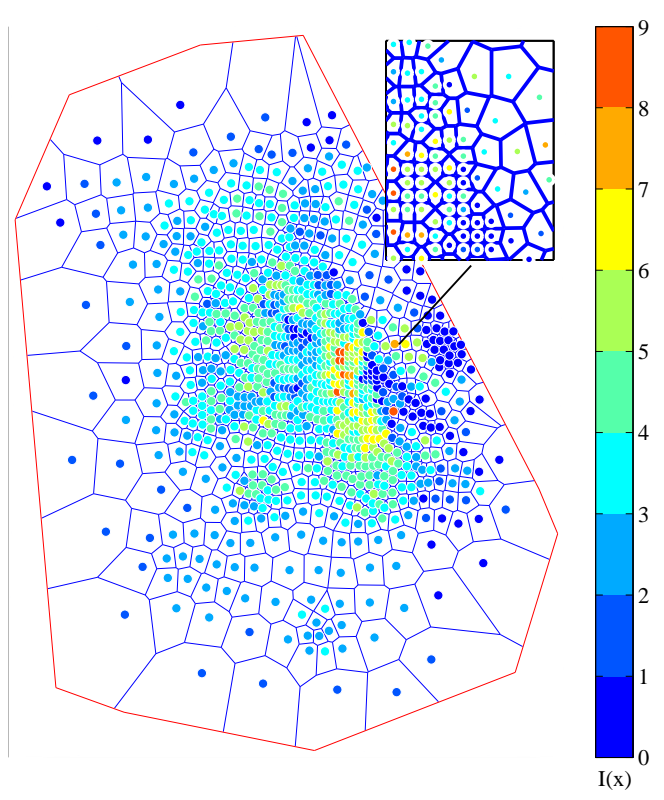

口

$$
\text { (b) } h=\$ 91 / \mathrm{EV}
$$

Figure 11: Chicago Case: Optimal station locations and corresponding EV fleet sizes with varying $h$. 
Table 5: Relative cost errors and solution times under the PTA criterion for the Chicago network.

\begin{tabular}{|c|c|c|c|c|c|c|c|c|c|}
\hline \multirow{2}{*}{ Parameter } & \multirow{2}{*}{ Value } & \multicolumn{6}{|c|}{ Relative errors } & \multicolumn{2}{|c|}{ Solution times (secs) } \\
\hline & & $\bar{C}$ & $\bar{C}_{f}$ & $\bar{C}_{w}$ & $\bar{C}_{h}$ & $\bar{C}_{b}$ & $\bar{C}_{d}$ & $\mathrm{CA}$ & Discretization \\
\hline \multicolumn{2}{|c|}{ Benchmark } & $-0.1 \%$ & $0.0 \%$ & $\overline{-2.0 \%}$ & $2.5 \%$ & $4.3 \%$ & $\overline{0.2 \%}$ & 26.2 & 585.8 \\
\hline \multirow{2}{*}{$f$} & $\$ 100$ & $-0.8 \%$ & $0.0 \%$ & $-6.0 \%$ & $5.1 \%$ & $-1.7 \%$ & $0.1 \%$ & 26.5 & 608.4 \\
\hline & $\$ 1000$ & $-0.8 \%$ & $0.1 \%$ & $-3.7 \%$ & $4.8 \%$ & $19.0 \%$ & $0.3 \%$ & 30.3 & 621.7 \\
\hline \multirow{2}{*}{$w$} & $\$ 1.25 / \mathrm{km}$ & $2.8 \%$ & $0.0 \%$ & $-3.9 \%$ & $5.9 \%$ & $15.7 \%$ & $0.2 \%$ & 31.0 & 612.1 \\
\hline & $\$ 12.5 / \mathrm{km}$ & $11.4 \%$ & $0.0 \%$ & $18.2 \%$ & $1.4 \%$ & $-4.1 \%$ & $0.2 \%$ & 26.5 & 594.5 \\
\hline \multirow{2}{*}{$h$} & $\$ 9.1 /$ veh & $9.1 \%$ & $0.0 \%$ & $18.4 \%$ & $1.3 \%$ & $0.2 \%$ & $0.2 \%$ & 27.5 & 594.9 \\
\hline & $\$ 91 /$ veh & $3.41 \%$ & $0.1 \%$ & $5.5 \%$ & $1.9 \%$ & $6.6 \%$ & $0.2 \%$ & 29.3 & 618.9 \\
\hline \multirow{2}{*}{$b$} & $\$ 10 /$ veh & $11.2 \%$ & $0.0 \%$ & $23.4 \%$ & $1.3 \%$ & $3.0 \%$ & $0.1 \%$ & 28.8 & 596.6 \\
\hline & $\$ 40 /$ veh & $9.7 \%$ & $0.1 \%$ & $20.3 \%$ & $1.4 \%$ & $3.2 \%$ & $0.2 \%$ & 25.5 & 581.0 \\
\hline \multirow{2}{*}{$v$} & $20 \mathrm{~km} / \mathrm{h}$ & $-1.5 \%$ & $0.0 \%$ & $-14.5 \%$ & $2.5 \%$ & $-0.1 \%$ & $0.1 \%$ & 23.2 & 607.9 \\
\hline & $80 \mathrm{~km} / \mathrm{h}$ & $-0.9 \%$ & $0.0 \%$ & $-11.6 \%$ & $2.4 \%$ & $-0.1 \%$ & $0.1 \%$ & 23.5 & 593.1 \\
\hline \multirow{2}{*}{$\bar{s}$} & $80 \mathrm{~km}$ & $-0.8 \%$ & $0.0 \%$ & $-12.7 \%$ & $2.8 \%$ & $0.4 \%$ & $0.1 \%$ & 22.9 & 414.3 \\
\hline & $320 \mathrm{~km}$ & $-0.5 \%$ & $0.0 \%$ & $-9.2 \%$ & $2.3 \%$ & $-0.3 \%$ & $0.0 \%$ & 23.0 & 296.5 \\
\hline \multirow{2}{*}{$\bar{r}$} & $3.5 \mathrm{~h}$ & $-1.2 \%$ & $0.0 \%$ & $-13 \%$ & $2.5 \%$ & $-0.1 \%$ & $0.0 \%$ & 23.2 & 593.1 \\
\hline & $14 \mathrm{~h}$ & $-0.9 \%$ & $0.0 \%$ & $-11 \%$ & $2.4 \%$ & $-0.1 \%$ & $0.1 \%$ & 23.0 & 589.8 \\
\hline \multicolumn{2}{|c|}{ Mea $5 \%$ n (of absolute values) } & $3.7 \%$ & $0.02 \%$ & $11.6 \%$ & $2.7 \%$ & $3.9 \%$ & $0.14 \%$ & 26.0 & 567.2 \\
\hline
\end{tabular}

